\title{
Review Article \\ Recent Fuzzy Generalisations of Rough Sets Theory: A Systematic Review and Methodological Critique of the Literature
}

\author{
Abbas Mardani, ${ }^{1}$ Mehrbakhsh Nilashi, ${ }^{2,3}$ Jurgita Antucheviciene, \\ Madjid Tavana, ${ }^{5,6}$ Romualdas Bausys, ${ }^{7}$ and Othman Ibrahim ${ }^{2}$ \\ ${ }^{1}$ Faculty of Management, Universiti Teknologi Malaysia (UTM), 81310 Skudai, Johor, Malaysia \\ ${ }^{2}$ Faculty of Computing, Universiti Teknologi Malaysia, 81310 Skudai, Johor, Malaysia \\ ${ }^{3}$ Department of Computer Engineering, Lahijan Branch, Islamic Azad University, Lahijan, Iran \\ ${ }^{4}$ Department of Construction Management and Real Estate, Vilnius Gediminas Technical University, \\ Sauletekio Al. 11, LT-10223 Vilnius, Lithuania \\ ${ }^{5}$ Business Systems and Analytics Department, La Salle University, Philadelphia, PA 19141, USA \\ ${ }^{6}$ Business Information Systems Department, Faculty of Business Administration and Economics, \\ University of Paderborn, 33098 Paderborn, Germany \\ ${ }^{7}$ Department of Graphical Systems, Vilnius Gediminas Technical University, Sauletekio Ave. 11, LT-10223 Vilnius, Lithuania
}

Correspondence should be addressed to Jurgita Antucheviciene; jurgita.antucheviciene@vgtu.lt

Received 12 April 2017; Revised 23 August 2017; Accepted 10 September 2017; Published 29 October 2017

Academic Editor: Danilo Comminiello

Copyright (C) 2017 Abbas Mardani et al. This is an open access article distributed under the Creative Commons Attribution License, which permits unrestricted use, distribution, and reproduction in any medium, provided the original work is properly cited.

\begin{abstract}
Rough set theory has been used extensively in fields of complexity, cognitive sciences, and artificial intelligence, especially in numerous fields such as expert systems, knowledge discovery, information system, inductive reasoning, intelligent systems, data mining, pattern recognition, decision-making, and machine learning. Rough sets models, which have been recently proposed, are developed applying the different fuzzy generalisations. Currently, there is not a systematic literature review and classification of these new generalisations about rough set models. Therefore, in this review study, the attempt is made to provide a comprehensive systematic review of methodologies and applications of recent generalisations discussed in the area of fuzzy-rough set theory. On this subject, the Web of Science database has been chosen to select the relevant papers. Accordingly, the systematic and metaanalysis approach, which is called "PRISMA," has been proposed and the selected articles were classified based on the author and year of publication, author nationalities, application field, type of study, study category, study contribution, and journal in which the articles have appeared. Based on the results of this review, we found that there are many challenging issues related to the different application area of fuzzy-rough set theory which can motivate future research studies.
\end{abstract}

\section{Introduction}

Rough set theory is a powerful and popular machine learning method [1]. It is particularly appropriate for dealing with information systems that exhibit inconsistencies [2]. Fuzzyrough set theory can be integrated with the rough set theory to handle data with continuous attributes and can detect inconsistencies in the data. Because the fuzzy-rough set model is a powerful tool in analysing inconsistent and vague data, it has proven to be very useful in many application areas.
Rough set theory, introduced by Pawlak [3] in the 1980s, is a powerful machine learning tool that has applications in many data mining [4-11] instances, attribute and feature selection [12-25], and data prediction [26, 27]. Rough set theory deals with information systems that contain inconsistent data, such as two patients who have the same symptoms but different diseases. In the rough set analysis, data is expected to be discrete. Therefore, a continuous numeric attribute is required to be discretized. Fuzzy-rough set theory [28] is an extension of the rough set theory that deals with continuous numerical 
attributes. It can solve the same problems that rough set can solve and also can handle both numerical and discrete data. The importance of fuzzy-rough set theory is clearly seen in several applications areas. For example, Wang [29] and Wang [30] investigated topological characterizations of generalised fuzzy-rough sets in the context of basic rough equalities. Pan et al. [31] enhanced the fuzzy preference relation rough set model with an additive consistent fuzzy preference relation. Namburu et al. [32] suggested the soft fuzzy-rough set-based magnetic resonance brain image segmentation for handling the uncertainty related to indiscernibility and vagueness. $\mathrm{Li}$ et al. [33] proposed an effective fuzzy-rough set model for feature selection. Feng et al. [34] used uncertainty measures for reduction of multigranulation based on fuzzy-rough sets, avoiding the negative and positive regions. Sun et al. [35] presented three kinds of multigranulation fuzzy-rough sets over two universes using a constructive method. Zhao and $\mathrm{Hu}$ [36] examined a decision-theoretic rough set model in the context of models of interval-valued fuzzy and fuzzy probabilistic approximation spaces. Zhang and Shu [37] suggested a new paradigm based on generalised interval-valued fuzzy-rough sets by combining the theory of rough sets and theory of interval-valued fuzzy sets based on axiomatic and constructive methods. Zhang et al. [24] proposed a new fuzzy-rough set theory based on information entropy for feature selection. Wang and $\mathrm{Hu}$ [38] proposed arbitrary fuzzy relations by integrating granular variable precision fuzzy-rough sets and general fuzzy relations. Vluymans et al. [19] suggested a new kind of classifier for imbalanced multi-instance data based on fuzzy-rough set theory. Feng and Mi [39] investigated and reviewed the variable precision of multigranulation fuzzy decision-theoretic rough sets in an information system. Wang and $\mathrm{Hu}[40]$ presented novel generalised $L$-fuzzy-rough sets for generalisation of the notion of $L$-fuzzy-rough sets.

In recent decades, various kinds of models have been proposed and developed regarding the fuzzy generalisation of rough set theory. However, the literature review has not kept pace with the rapid addition of knowledge in this field. Therefore, we believe that there is a need for a systematic consideration of the most relevant recent studies conducted in this area. This review paper attempts to systematically review the previous studies that proposed or developed fuzzyrough sets theory. This review paper adds significant insight into the literature of fuzzy-rough set theory, by considering some new perspectives in examining the articles, such as the classification of the papers based on author and year of publication, author nationalities, application field, type of study, study category, study contribution, and the journals in which they appear.

The structure of this review study is organised as follows. Section 2 shortly reviews the literature regarding fuzzy logic and fuzzy sets, rough sets, fuzzy set theory, fuzzy logical operators, fuzzy relations, rough set theory, and fuzzy-rough set theory. In Section 3, we present the related works. Section 4 presents research methodology including the systematic review, meta-analysis, and the procedures of this study. Section 5 provides findings of this review based on the application areas. Section 6 presents the distribution of papers by the journals. In Section 7, we present the distribution of papers by the year of publication. Section 8 presents the distribution of papers based on the nationality of authors. Section 9 discusses the results of this review with the focus on the recent fuzzy generalisation of rough sets theory and further investigations in this area. Finally, Section 10 presents the conclusion, limitations, and recommendations for future studies.

\section{Fuzzy-Rough Sets Theory}

2.1. Fuzzy Logic and Fuzzy Sets. Binary logic is discrete and has only two logic values which are true and false, that is, 1 and 0 . In real-life, however, things are true to some extent. For example, regarding the patient there are some assumptions such as patient is sick, or say patient is very sick or starting to become sick. Therefore, we cannot confidently say if the patient is sick or not with a certain intensity. Fuzzy logic [43] has extended binary logic through adding an intensity range of values to specify the extent to which something is true. In this situation, the range of truth values is between 0 and 1 . The closer the truth value of a statement to 1 , the truer the statement. For example, the patient is very sick could have the degree of sickness around 0.9 to specify that the patient is very sick. On the other side, patient could have the sickness degree of 0.1 indicating that the patient is nearly recovered from the illness. A fuzzy set [44] is a set of factors that fit to the set with the membership degree. For instant, we assume that there are two fuzzy sets representing two groups of people including old and young people. Thus the larger the age of one person, the higher the membership degree to the old people, and the lower the person membership degree to the young people. Meanwhile fuzzy logic is extending the binary logic and moreover extends its logical operations. These are the $t$ conorm fuzzy logical $t$-norm and implicator [45] that extend the binary logical implication conjunction and disjunction.

2.2. Rough Sets. In the very big datasets with several items, calculating the gradual indiscernibility relation is very challenging in terms of memory and runtime. Rough set theory (Pawlak [46], Polkowski et al. [47]) is the novel mathematics technique dealing with uncertain and inexact knowledge in several applications in various real-life fields such as information analysis, medicine, and data mining. Rough set is the powerful machine learning technique which has been used in many application areas such as feature selection, prediction, instance selection, and decision-making. Rough set also has applications in many areas such as medical data analysis, image processing, finance, and many other real-life problems [3]. A rough set approximates a certain set of factors with two subsets including upper approximations and lower approximations. The fuzzy-rough set theory is constructed based on two theories including rough set theory and fuzzy set theory. In the next section, we present these theories with hybridisation of both theories.

2.3. Fuzzy Set Theory. Zadeh [44] found that traditional crisp set is not capable of explaining the whole thing in the real manner. Zadeh proposed fuzzy sets to solve this problem. Therefore Zadeh proposed a fuzzy set $A$ as a mapping from 
the universe $U$ to the interval $[0,1]$. The set $A(x)$ for $x \in U$ is called the degree of membership of $x$ in $A$. Employ this method; factors in the universe could belong to a set to a certain degree. Discovery for the good fuzzy set for model concepts could be subjective and challenging; however it is more significant than trying to create an artificial crisp distinction among factors. Indeed that fuzzy set was an extension of crisp set. Consequently, any crisp set $A$ could be modelled using a fuzzy set as follows:

$$
\begin{aligned}
& \forall x \in U: A_{(x)}=\left\{\begin{array}{l}
1 \\
0
\end{array}\right. \\
& \forall x \in U: A_{(x)}= \begin{cases}1 & \text { if } x \in A \\
0 & \text { else. }\end{cases}
\end{aligned}
$$

The cardinality of a fuzzy set $A$ is defined as the sum of the membership values of all factors in the universe to $A$ :

$$
|A|=\sum_{x \in U} A_{(x)} .
$$

2.4. Fuzzy Logical Operators. There is need for the new logical operators to extend the crisp sets to the fuzzy sets. In crisp set theory, for example, the proposition a factor belongs to the sets $A$ and $B$ is either true or false. For extending this theorem to fuzzy set theory, there is need to the fuzzy logical operators for extending the logical conjunction $\wedge$, for expressing to what extent an example $x$ belongs to $A$ and $B$, given the membership degrees $A(x)$ and $B(x)$.

The conjunction $\wedge$ and the disjunction $\vee$ were extended by using the $t$-conorm $\delta$ and $t$-norm $\mathscr{T}$ that map $\mathscr{T}, S$ : $[0,1]^{2} \rightarrow[0,1]$, satisfying the following conditions:

$$
\begin{aligned}
& \mathcal{T} \text { and } \mathcal{S} \text { are increasing in both arguments. } \\
& \mathscr{T} \text { and } \mathcal{S} \text { are commutative. } \\
& \mathscr{T} \text { and } \mathcal{S} \text { are associative. }
\end{aligned}
$$

$$
\begin{aligned}
& \forall x \in U: \mathscr{T}(x, 1)=x, \\
& \forall x \in U: \delta(x, 0)=x .
\end{aligned}
$$

The most significant examples of $t$-norms are the minimum operator $\tau_{M}$, which is the largest $t$-norm, the product operator $\mathscr{T}_{P}$, and the Łukasiewicz $t$-norm $\mathscr{T}_{L}$ :

$$
\begin{aligned}
& \forall x, y \in(0,1): \mathscr{T}_{M}(x, y)=\min (x, y) \\
& \forall x, y \in(0,1): \mathscr{T}_{P}(x, y)=x y \\
& \forall x, y \in(0,1): \mathscr{T}_{L}(x, y)=\max (0, x+y-1) .
\end{aligned}
$$

The important examples of $t$-conorms are the maximum operator $\mathcal{S}_{M}$, which is the smallest $t$-conorm, the probabilistic sum $\mathcal{S}_{p}$, and the Eukasiewicz $t$-conorm $\mathcal{S}_{L}$ :

$$
\begin{aligned}
& \forall x, y \in(0,1): \mathcal{S}_{M}(x, y)=\max (x, y) \\
& \forall x, y \in(0,1): \mathcal{S}_{p}(x, y)=x+y-x y \\
& \forall x, y \in(0,1): \mathcal{S}_{L}(x, y)=\min (1, x+y) .
\end{aligned}
$$

The implication $\rightarrow$ is extended by fuzzy implicators, which are mappings $\mathfrak{T}:[0,1]^{2} \rightarrow[0,1]$ that satisfy the following:

$L$ is decreasing in the first and increasing in the second argument.

$$
L \text { satisfies } L(1,1)=L(0,0)=1 \text { and } L(1,0)=0 .
$$

The well-knowing implicator is the Eukasiewicz implicator $L_{L}$, defined by

$$
\forall x, y \in(0,1): L_{L}(x, y)=\min (1,1-x+y) .
$$

2.5. Fuzzy Relations. The binary fuzzy relations in $U$ are the special type of fuzzy sets which are fuzzy sets $R$ in $U^{2}$ and express to what extent $x$ and $y$ are associated with others. In the field of fuzzy-rough set, usually use relations for modelling indiscernibility between the examples. Therefore, we refer to them as indiscernibility relations. We need $R$ to be minimum a fuzzy tolerance relation; that is, $R$ is reflexive: $\forall x \in U, R(x, x)=1$ and symmetric $\forall x, y \in U, R(x, y)=$ $R(y, x)$.

These two situations are linked to the symmetry and reflexivity conditions of the equivalence relation. The third situation for an equivalence relation, transitivity, is translated to $\mathscr{T}$-transitivity for a certain $t$-norm $\mathscr{T}$ :

$$
\forall x, y, z \in \mathscr{T}(R(x, y), R(y, z)) \leq R(x, z) .
$$

For this case, $R$ is called a $\mathscr{T}$-similarity relation. Thus when $R$ is $\mathscr{T}_{M}$-transitive, $R$ is $\mathscr{T}$-transitive for all $t$-norms $\mathscr{T}$. For this case, $R$ will be a similarity relation.

2.6. Rough Set Theory. Pawlak [3] proposed the rough set theory for handling the problem of incomplete information. Pawlak introduced a universe $U$ involving factors, an equivalence relation $R$ on $U$, and a concept $A \subseteq U$ within the universe. The problem of incomplete information indicates that it should not be possible to determine the concept $A$ based on the equivalence relation $R$, which is there are two factors $x$ and $y$ in $U$ that are equivalent to $R$ but to which $x$ belongs to $A$ and $y$ does not. Figure 1 represented this case in which the universe is divided into squares applying the equivalence relation. The concept $A$ does not follow the lines of the squares, which means that $A$ cannot be described using $R$. In real world this kind of problem with incomplete information often occurs such as problem of spam classification. For example we assume the world contains nonspam and spam e-mails and mention that this concept is spam. The equivalence relation is introduced according to the predefined list of 10 words which usually are in the spam e-mails; thus it can mention that two emails are equivalent if they have the similar words among the list of 10 words. Some equivalence classes will be completely contained in the spam group, and some will be completely contained in the nonspam group. Though it is very likely that there are two e-mails that have the similar words among the list of 10 words, however for which one is spam and the other is nonspam. In this case, the equivalence relation is not 


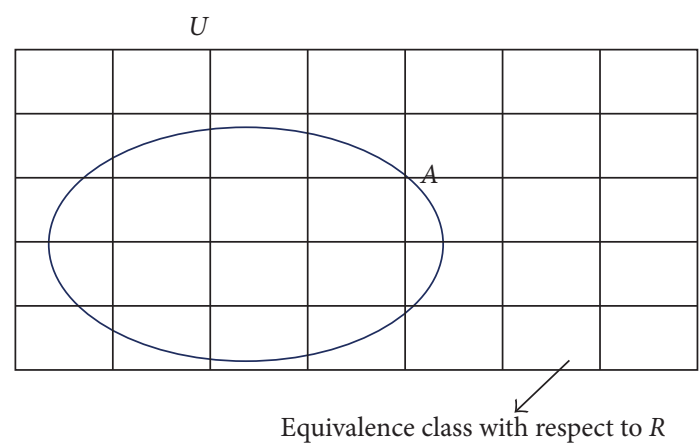

FIgURE 1: A universe $U$ partitioned by an equivalence relation $R$ and a concept $A \subseteq U$ that cannot be defined using $R[41,42]$.

able to distinguish between spam and nonspam. Pawlak [3] addressed this kind of problem by approximating the concept $A$. The lower approximation includes all the equivalence classes that are included in $A$, and the upper approximation includes the equivalence classes for which at least one factor is in A. Figure 2 represented the concept of lower and upper approximations.

An equivalence relation is reflexive; thus $x$ is only included in $R \downarrow A$ if $x \in A$. The upper approximation is defined by

$$
R \uparrow A=\left\{x \in U \mid \exists_{y} \in U:(x, y) \in R \wedge y \in A\right\} .
$$

The lower approximation of $A$ using $R$ is defined as follows:

$$
R \downarrow y=\{x \in U \mid \forall y \in U:(x, y) \in R \longrightarrow y \in A\} .
$$

The lower approximation in the spam example contains all e-mails that are spam and for which all e-mails indiscernible from it are also spam. The upper approximation contains of e-mails that are spam and e-mails that are nonspam but for which there exists an e-mail indiscernible from it that is spam.

2.7. Fuzzy-Rough Set Theory. Fuzzy set theory enables us to model vague information, while rough set theory models incomplete information. These two theories are not competing, but rather complement each other. Many models have been proposed to hybridise rough sets and fuzzy sets $[6,29,30,34,39,170-178]$. A fuzzy-rough set is the pair of lower and upper approximations of a fuzzy set $A$ in a universe $U$ on which a fuzzy relation $R$ is defined. The fuzzy-rough model is obtained by fuzzifying the definitions of the crisp lower and upper approximation. Recall that the condition for an element $x \in U$ to belong to the crisp lower approximation is

$$
\forall y \in U(x, y) \in R \longrightarrow y \in A .
$$

The equivalence relation $R$ is now a fuzzy relation, and $A$ is a fuzzy set. The values $R(x ; y)$ and $A(y)$ are connected by a fuzzy implication $\mathscr{L}$, so $\mathscr{L}(R(x ; y), A(y))$ expresses to what extent elements that are similar to $x$ belong to $A$.
The membership value of an element $x \in U$ to the lower approximation is high if these values $\mathscr{L}(R(x ; y), A(y))$ are high for all $y \in A$ :

$$
\begin{aligned}
& \forall x \in U(R \downarrow A)(x)=\min _{y \in U} \mathscr{L}(R(x, y), A(y)) . \\
& \forall x \in U(R \uparrow A)(x)=\max _{y \in U} \mathscr{T}(R(x, y), A(y)) .
\end{aligned}
$$

This upper approximation expresses to what extent there exist instances that are similar to $x$ and belong to $A$.

\section{Related Works}

Morsi and Yakout [179] examined the relationship between fuzzy-rough sets theory based on $R$-implicators and leftcontinuous $t$-norms, with focus on fuzzy similarity in the axiomatic approach. Wang and Hong [180] proposed an algorithm to produce a set of fuzzy rules from noisy quantitative training data, by applying the variable precision rough set model. Molodtsov [181] employed the theory of rough sets in several ways and formulated the soft number notions, soft integral and soft derivative. Maji et al. [182] examined the soft sets in detail and provided the application of soft sets in decision-making by employing the rough sets reduction. Radzikowska and Kerre [28] investigated the family of fuzzyrough sets based on fuzzy implicators, which were called generalised fuzzy-rough sets. De Cock et al. [183] introduced fuzzy-rough sets based on $R$-foresets of all objects with respect to a fuzzy binary relation, when $R$ is a fuzzy serial relation. Jensen and Shen [184] proposed classical rough sets based on a dependency function of fuzzy-rough sets and presented the new greedy algorithm for reduction of redundant attributes. Mieszkowicz-Rolka and Rolka [185] proposed an approach based on the variable precision fuzzyrough set approach to the analysis of noisy data. Mi and Zhang [186] introduced a new fuzzy-rough set definition based on a residual implication, $\theta$ and its dual, $\sigma$. Shen and Jensen [187] proposed an approach that integrates a fuzzy rule induction algorithm with a fuzzy-rough method for feature selection. Wu et al. [188] examined generalised fuzzy-rough sets in the axiomatic method. Bhatt and Gopal [189] proposed a new concept of compact computational domain for Jensen's algorithm for enhancing computational efficiency. Yeung et al. [190] proposed some fuzzy-rough set models by means of arbitrary fuzzy relations and investigated the connections between the existing fuzzy-rough sets. Deng et al. [191] investigated fuzzy relations by involving a fuzzy covering. Li and Ma [192] proposed two pairs of fuzzy-rough approximation operators, including fuzzy and crisp covering-based fuzzyrough approximation operators. Aktaş and Çağman [193] compared the concepts of rough sets, soft sets, and fuzzy sets and showed that, for each fuzzy set, rough set is a soft set. Greco et al. [194] integrated the DTRS approach with the dominance-based rough set approach and presented a new generalised rough set theory approach. Cornelis et al. [195] introduced a classical rough set approach by utilising fuzzy tolerance relations, considering fuzzy-rough set theory. Wang et al. [196] introduced new definitions of fuzzy lower 


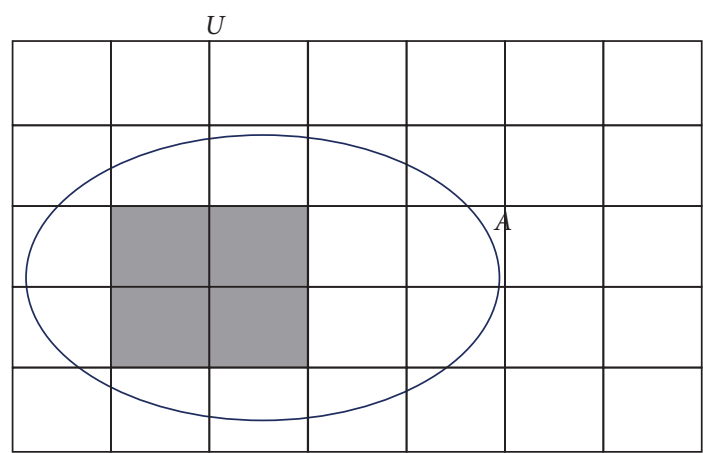

(a) The lower approximation

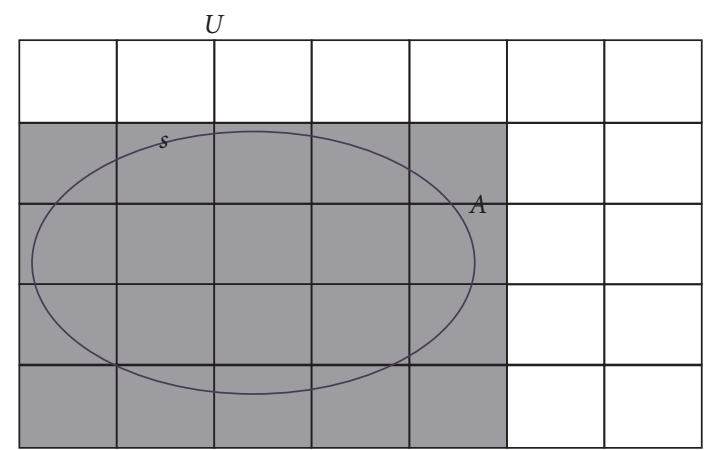

(b) The upper approximation

FIgURE 2: The concept $A$ is approximated by means of the lower and upper approximations [41, 42].

and upper approximations by using the similarity between two objects. Hu et al. [197] proposed a novel fuzzy-rough set model, based on which a straightforward and efficient hybrid attribute reduction algorithm was designed. Lingras et al. [198] and Lingras et al. [199] applied the DTRS theory for clustering analysis. She and Wang [200] investigated $L$ fuzzy-rough set theory from an axiomatic method. Zhao et al. [201] suggested the new concept based on fuzzy variable precision rough sets for handling the noise of perturbation and misclassification. Wu et al. [202] introduced a new attribute reduction approach, focusing on the interval type 2 fuzzy-rough set model and providing some properties of interval type 2 fuzzy-rough sets. Yan et al. [203] proposed generalising the rough set model with fuzzy relation on two universes using fuzzy theory and probabilistic methods. Yang and Yao [204] investigated the multiagent DTRS model. Xu et al. [205] examined the GRS approach based on the rough membership function. Xia and $\mathrm{Xu}$ [206] investigated intuitionistic fuzzy information changing. Wu [207] examined fuzzy-rough sets based on $t$-norms and discussed algebraic structures and fuzzy topologies. Feng et al. [208] suggested the soft rough sets concept instead of equivalence classes and defined the parameterized soft set of upper and lower approximations. Meng et al. [209] suggested an approach for calculating the upper and lower approximations for fuzzy soft sets which efficiently define the boundary. Li and Zhou [210] and $\mathrm{Li}$ et al. [211] suggested a multiperspective explanation of the decision-theoretic rough set approach and attribute reduction. Jia et al. [212] investigated the problem related to attribute reduction for decision-theoretic rough set theory. Liu et al. [213] and Liu et al. [214] explored multiple-category classification with the DTRS method and applications in management science. Degang et al. [215] introduced a novel approach to examine fuzzy-rough sets by integrating granular computing. Hu et al. [94] used kernel functions for introducing the fuzzy similarity relations and developed the greedy algorithm based on dimensionality reduction. Ma and Sun [216] proposed a probabilistic rough set model on two universes. Ma and Sun [217] investigated the decisiontheoretic rough set theory over two universes, based on the idea of classical DTRS theory. Liu et al. [218] studied the GRS approach based on two universes and discussed its properties. Chen et al. [102] used fuzzy-rough sets to present a matrix of fuzzy discernibility. Zhang et al. [111] proposed a general framework of intuitionistic fuzzy-rough sets and discussed the intuitionistic fuzzy operator and the properties of the model with the dual domain case. Wei et al. [219] investigated the relationships among rough approximations of fuzzyrough set models. Chen et al. [117] explored the interpretation of several types of membership functions, geometrically, by using the lower approximations in fuzzy-rough sets, in terms of square distances in Krein spaces. $\mathrm{Ma}$ and $\mathrm{Hu}$ [220] examined lattice structures and the topology of $L$-fuzzyrough sets by considering upper and lower approximations sets. Yang et al. [70] suggested a fuzzy probabilistic rough set model on two universes. Liang et al. [59] examined information retrieval and filtering by employing the DTRS theory. Zhang and Miao [221] and Zhang and Miao [222] proposed the double-quantitative approximation space for presenting two double-quantitative rough set theories. Yao et al. [101] suggested the variable precision $(\theta, \sigma)$-fuzzyrough sets theory regarding fuzzy granules. Liu et al. [223] investigated logistic regression for classification based on decision-theoretic rough sets theory. Ma et al. [224] suggested a decision region distribution preservation reduction in decision-theoretic rough set theory. Qian et al. [225] investigated the multigranulation decision-theoretic rough set approach. Sun et al. [82] examined the DTRFS application and model. Zhang and Miao [226] constructed a fundamental reduction framework for two-category decision-theoretic rough sets. Gong and Zhang [105] investigated a method of intuitionistic fuzzy sets and variable precision rough sets, to construct an extended intuitionistic fuzzy-rough set model. Zhao and Xiao [131] defined the general type-2 fuzzy-rough sets and discussed the basic properties of lower and upper approximation operators. Zhang and Miao [227] and Zhang and Miao [228] examined attribute reduction for proposing rough set theory models. D'eer et al. [118] investigated the drawbacks and benefits of implicator-conjunctor-based noise-tolerant fuzzy-rough set models. Li and Cui [77] studied the characterizations of the topology of fuzzy-rough sets by considering the similarity of fuzzy relations. $\mathrm{Li}$ and Cui [229] investigated fuzzy topologies considering a lower fuzzy-rough approximation operator based on the $t$-conorm. $\mathrm{Xu}$ et al. [230] introduced a knowledge reduction approach in a generalised approximation space over two universes. Li and 
$\mathrm{Xu}$ [231] introduced the multigranulation decision-theoretic rough set approach in the ordered information system. Liang et al. [232] suggested three-way decisions by extending the DTRS approach to a qualitative environment. Ju et al. [233] presented a moderate attribute reduction model in DTRS. Li and Xu [234] investigated the double-quantitative DTRS approach based on assembling the upper and lower approximations of the GRS and DTRS methods. Wang [114] examined type 2 fuzzy-rough sets by considering two finite universes and utilising axiomatic and constructive methods and investigated some topological properties of type 2 fuzzyrough sets. Zhang and Min [235] used three-way decisions regarding recommender systems. Wang [114] defined an upper approximation number for developing a quantitative analysis of covering-based rough set theory. Wang et al. [25] proposed a fitting fuzzy-rough set model to conduct feature selection. Yang and $\mathrm{Hu}$ [178] proposed a definition of fuzzy $\beta$-covering approximation spaces by introducing some new definitions of fuzzy $\beta$-covering approximation spaces, Ma's fuzzy covering-based rough set and the properties of fuzzy $\beta$-covering approximation spaces. Wang [29] investigated characterizations of generalised fuzzy-rough sets in the core rough equalities context. Namburu et al. [32] suggested a soft fuzzy-rough set-based segmentation of magnetic resonance brain image for handling the uncertainty regarding the indiscernibility and vagueness in a parameterized representation. Wang [30] examined the topological structures of $L$-fuzzyrough set theory. Liang et al. [232] examined triangular fuzzy decision-theoretic rough sets. Zhang and Yao [236] examined functions of the Gini objective for three-way classification. Sun et al. [237] explored three-way group decision-making by using multigranulation fuzzy decision-theoretic rough sets. Feng et al. [34] investigated reduction of the multigranulation fuzzy information system based on uncertainty measures by considering variable precision multigranulation decisiontheoretic fuzzy-rough set theory and avoiding the changing of negative region and positive region to small ones. Sun et al. [62] proposed the new fuzzy-rough set on a probabilistic approximation space and used it with respect to decisionmaking in unconventional emergency management. $\mathrm{Hu}$ et al. [171] proposed a new incremental method to update approximations of fuzzy information system over two universes. Fan et al. [72] introduced several double-quantitative DTRS (DQ-DTRFS) models based on logical conjunction and disjunction operations. Qiao and $\mathrm{Hu}$ [238] proposed a granular variable precision $L$-fuzzy-rough set theory based on residuated lattices with arbitrary $L$-fuzzy relations. Liang et al. [172] examined the decision principles of three-way decision rules based on the variation of loss functions with IFSs.

\section{Research Method}

For the research methodology in this study, we used the Preferred Reporting Items for Systematic Reviews and MetaAnalyses (PRISMA) provided by Moher et al. [239]. PRISMA has two main parts, including systematic reviews and metaanalyses. Systematic reviews provide objective summaries of what has been written and found out about research topics.
This is especially valuable in wide research areas, where many publications exist, each focusing on a narrow aspect of the field [240]. Systematic reviews aim to provide a full overview of research conducted in a specific area until the present date. All research procedures have to be made explicit before the actual behaviour of the review to make the process objective and replicable. Meta-analysis provides a means of mathematically integrating findings employing diverse statistical approaches to study the diversity of the articles. In this kind of synthesis, original studies that are compatible with their quality level are selected. This aspect may help and highlight different facts which individual primary studies fail to do, for example, it may prove that results are statistically significant and relevant when small primary studies provide inconclusive and uncertain results with a large confidence interval [241]. The main goal of PRISMA is to help the researchers and practitioners complete a comprehensive and clear literature review [242].

Several previous studies have been conducted using PRISMA in the various fields to develop a comprehensive literature review [242-244]. In order to implement the PRISMA method in this study, we accomplish three main steps including literature search, choosing the eligible published papers, and extraction of data and summarising [237].

4.1. Literature Search. In this step, we have chosen the Web of Science database to provide a comprehensive application of fuzzy-rough set theories. The literature search was accomplished based on two keywords including rough set theories and fuzzy-rough set theories. We attempted to collect the current published papers from 2010 to 2016. In the first step of our search, we found 5648 scholarly papers related to the rough set theories and fuzzy-rough set theories which were extracted according to our strategy search. In the next step, we searched to find the papers which were published between 2010 and 2016 and checked the duplicate papers with redundant information. After this step, 296 papers were remaining. After removing 17 records due to duplication, we screened papers based on the titles and abstracts, and irrelevant papers were removed. In total, 193 potentially related papers remained (see Figure 3).

4.2. Articles Eligibility. In this step of the review, for the purpose of eligibility, we reviewed the full text of each article independently (which extracted from the last step). In the last step, we carefully identified the related articles to attain a consensus. Book chapters, unpublished working papers, editorial notes, master dissertations and doctoral theses, textbooks, and non-English papers were excluded. In the end, we selected 132 articles related to the fuzzy-rough sets, from 28 international scholarly journals between 2010 and 2016, which met our inclusion criteria. We selected the papers from 2010 , because of such a large number of papers published in this field.

4.3. Data Extraction and Summarising. In the final step of our methodology, after negotiation with other authors, some required information was collected, and, finally, 132 articles were reviewed and summarised. In Table 1, all the selected 


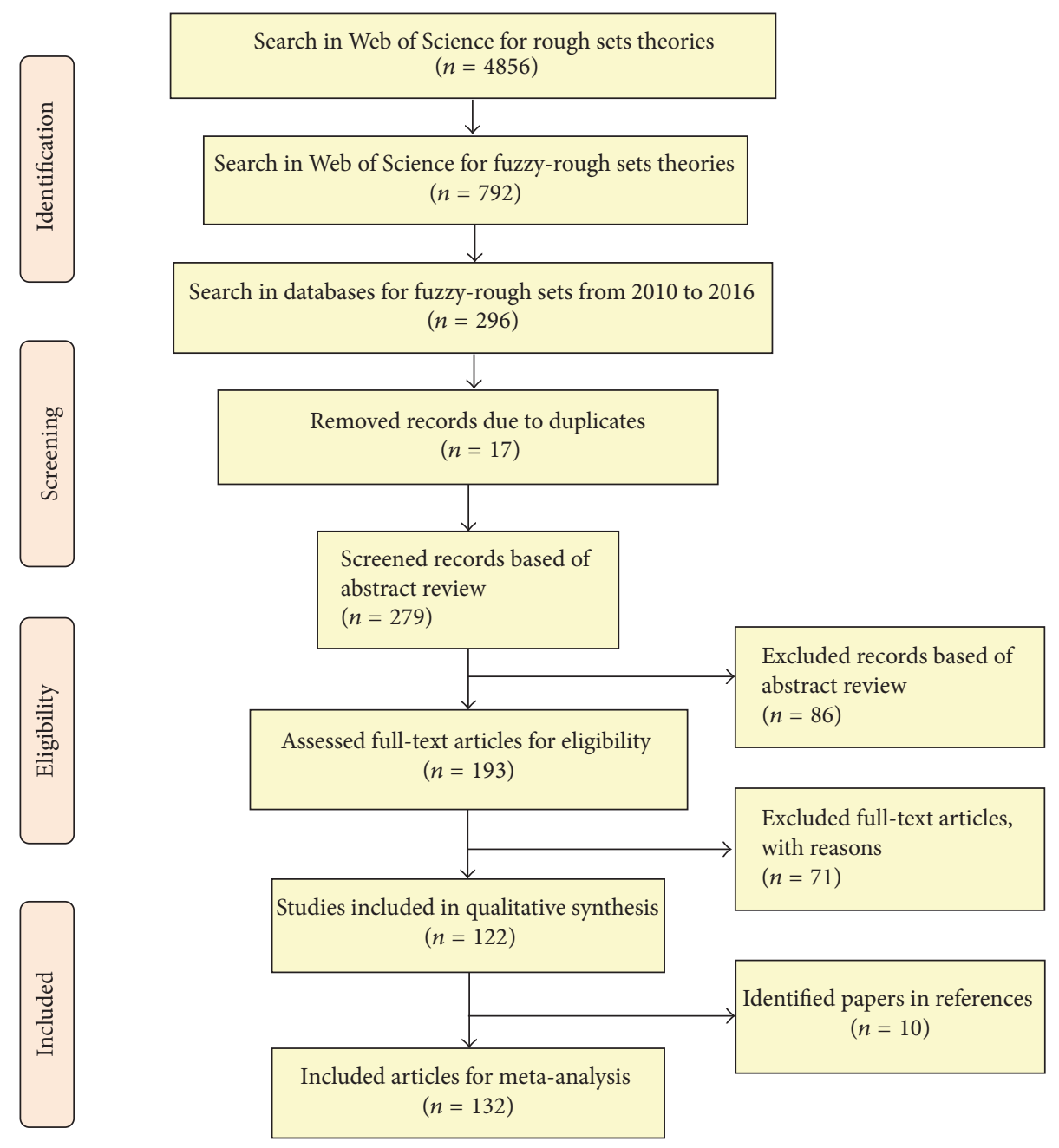

FIgURE 3: Study flowchart for the identification, screening, eligibility, and inclusion of articles.

TABLE 1: Classification of papers based on application area.

\begin{tabular}{lcc}
\hline Application areas & Frequency & Percentage of frequency \\
\hline Information systems & 12 & $9.09 \%$ \\
Decision-making & 9 & $8.33 \%$ \\
Approximation operators & 15 & $11.36 \%$ \\
Feature and attribute selection & 24 & $20.45 \%$ \\
Fuzzy set theories & 37 & $27.27 \%$ \\
Other application areas & 35 & $23.48 \%$ \\
\hline Total & 132 & $100.00 \%$ \\
\hline
\end{tabular}

articles were classified into different classifications including information systems, decision-making, approximate reasoning, feature and attribute selection, machine learning, fuzzy set theories, and other application areas. Also, the articles were summarised and reviewed based on the various criteria such as author and year of publication, author nationalities, application field, type of study, study category, study contribution, and journal in which they appeared. We believe that reviewing, summarising and classifying the articles helped us to achieve some critical and valuable insights. Consequently, some suggestions and recommendations for future studies were proposed. Furthermore, we believe that this review paper was accomplished very carefully and it presented a comprehensive source regarding the fuzzy-rough set theories. It should be noted that the main difficulty of using the PRISMA method was to understand what methodologies were used from the abstract and the research section of the selected articles. Thus, we required going through the full 
content of articles and took a more detailed look to evaluate the exactly applied approach to evaluate the fuzzy-rough sets. Although a considerable amount of time was spent in the selection process, it helped us choose the most suitable publications in conducting the review.

\section{Application Areas Classification}

Although categorising and combining the articles in the fields of fuzzy-rough sets is complex, for the classification task we used the opinions of experts. Consequently, based on opinions of experts we categorised articles into six different applications areas (see Table 1). In the following section, all selected articles were summarised and reviewed based on the various criteria.

5.1. Distribution Papers Based on Information Systems. Rough set theory is a powerful and popular machine learning method. It is particularly appropriate for dealing with the information systems that exhibit inconsistencies. Fuzzy set theory integrated with the rough set theory detects degrees of inconsistency in the data. Xu et al. [245] examined a new parallel attribute reduction algorithm by focusing on fuzzyrough set theory and mutual information rather than calculating of the fuzzy-rough lower and upper approximations explicitly. Moreover, Yang et al. [246] investigated some rough sets models for handling big data, but this study does not focus on explicitly calculating the upper and lower approximations. Some previous scholars investigated the parallel models for computing the upper and lower approximations in the traditional rough set model. For example, Zhang et al. [247] introduced a new MapReduce-based approach for calculating the upper and lower approximations in a decision system. Zhang et al. [248] used different approximations to compare knowledge acquisition. Yang and Chen [249] suggested a novel approach to calculate the positive region such as the union of the lower approximation of all classes. Dubois and Prade [250] explored the relationship, similarities, and differences between twofold fuzzy sets and rough sets. Ouyang et al. [50] defined new fuzzy rough sets which generalized the concept of fuzzy rough sets in the sense of Radzikowska and Kerre [28], and that of Mi and Zhang [186]. Kuznetsov et al. [251] introduced an approach based on the fuzzy-rough sets which were called the $(I, J)$-fuzzyrough set. Zeng et al. [52] developed a new Hybrid Distance (HD) in Hybrid Information System (HIS) based on the value difference metric, and a new fuzzy-rough approach was designed by integrating the Gaussian kernel and HD distance. Feng and $\mathrm{Mi}$ [39] investigated variable precision multigranulation fuzzy decision-theoretic rough sets in an information system. Chen et al. [55] introduced a new approach to build a polygonal rough-fuzzy set and presented a novel fuzzy interpolative reasoning approach for sparse fuzzy rule-based systems based on the ratio of fuzziness of the constructed polygonal rough-fuzzy sets. Table 2 represents significant distribution findings of information systems based on the author and year of publication, application field, type of study, study category, and study contribution. The results represented in this table indicate that 12 articles have been published in the area of information systems.

5.2. Distribution Papers Based on Decision-Making. While there are a variety of existing methods for various application areas from imprecise data, the fuzzy-rough set method has an advantage for decision-making for large volumes of data since it focuses on reducing the number of attributes required to characterize a concept without losing essential information required for decision-making. Although rough sets have several advantages over other methods, they generate a number of rules creating difficulties in decision-making [252]. A fuzzy-rough set is a data mining algorithm for decisionmaking based on incomplete, inconsistent, imprecise, and vague data. Fuzzy-rough set theory is an extension of the fuzzy conventional set theory that supports approximations in decision-making. However, rough set theories are valuable mathematical approaches for explaining and showing insufficient and incomplete information and also have been extensively used in numerous application areas such as comprehensive evaluation, and uncertainty decision-making with fuzzy information [62]. Greco et al. [253] proposed a new dominance rough set framework that is appropriate for preference analysis [31, 254-261]. This study investigated the decision-making problem with multicriteria and attributes, where dominance relations are extracted from similarity relations, and multicriteria are created from equivalence relations and numerical attributes are created from nominal attributes. An extensive review of multicriteria decision analysis based on dominance rough sets is given in Greco et al. [253]. Dominance rough sets have also been applied to ordinal attribute reduction $[253,262]$ and multicriteria classification [263-266]. Recently, several previous studies extended the fuzzy set based on the dominance rough set approach $[106,170,258,267,268]$. Hu et al. [60] proposed a novel approach for extracting the fuzzy preference relations by using a fuzzy-rough set model. Liang and Liu [61] presented a naive approach of intuitionistic fuzzy decision-theoretic rough sets (IFDTRSs) and analysed its related properties. Sun and $\mathrm{Ma}$ [65] introduced a novel concept of soft fuzzy-rough sets by integrating the traditional fuzzy-rough sets and fuzzy soft sets. The detailed results of this section are presented in Table 3. The findings represented in this table indicate that 8 articles have been published in the area of decision-making.

5.3. Distribution Papers Based on Approximation Operators. A rough set approximates a crisp set by two other sets that give a lower and upper approximation of the crisp set. In the rough set analysis, the data is expected to be discrete. In enormous information systems, the computation of the lower and upper approximation sets is a demanding process both regarding processing time and memory utilisation. A rough set approximates a certain set of elements with two other subsets called upper and lower approximations. Through the fuzzy-rough lower and upper approximation, fuzzy-rough set theory can model the quality or typicality of instances within their respective classes, and hence it is an ideal tool to detect border points and noisy instances. The lower approximation 


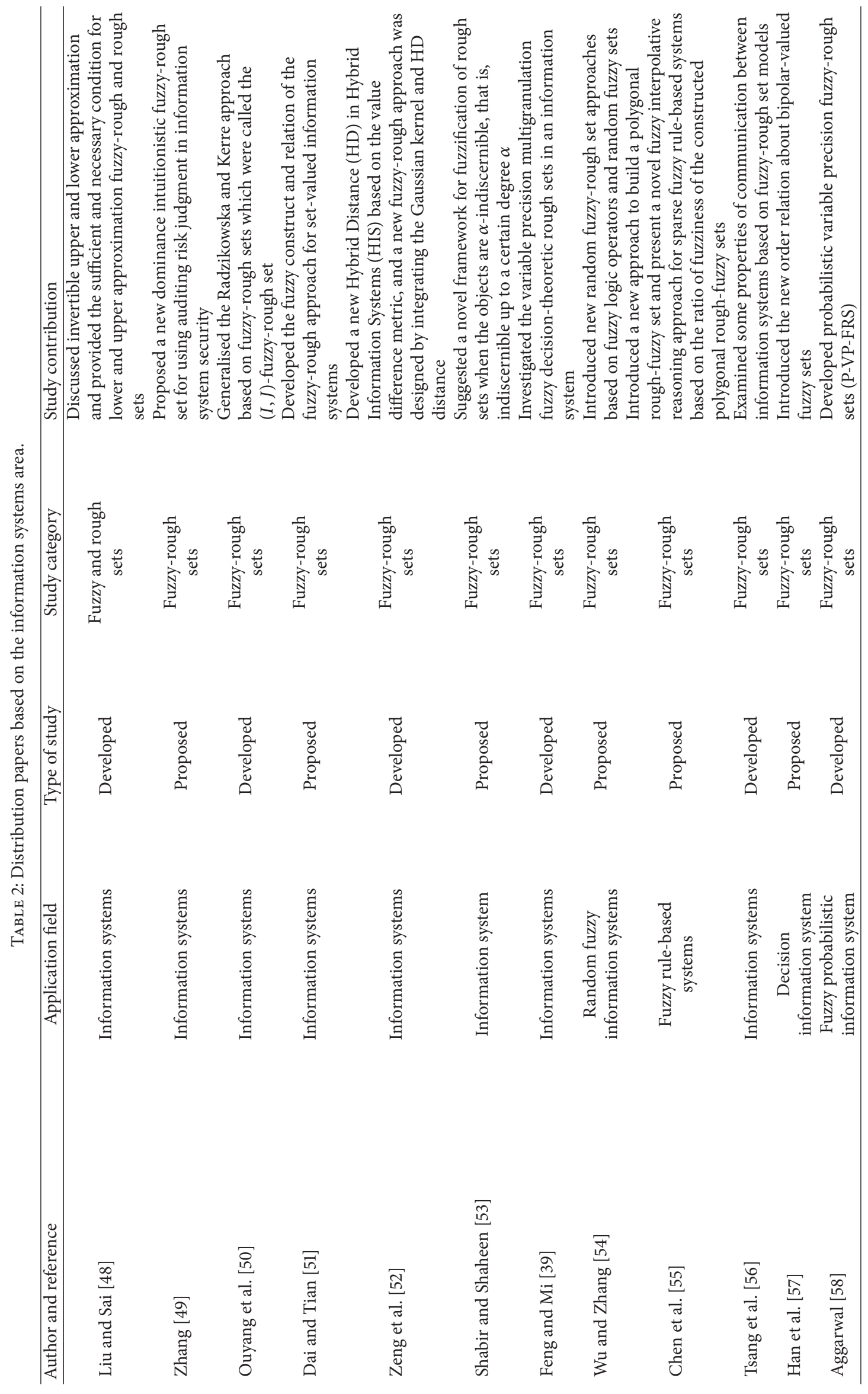




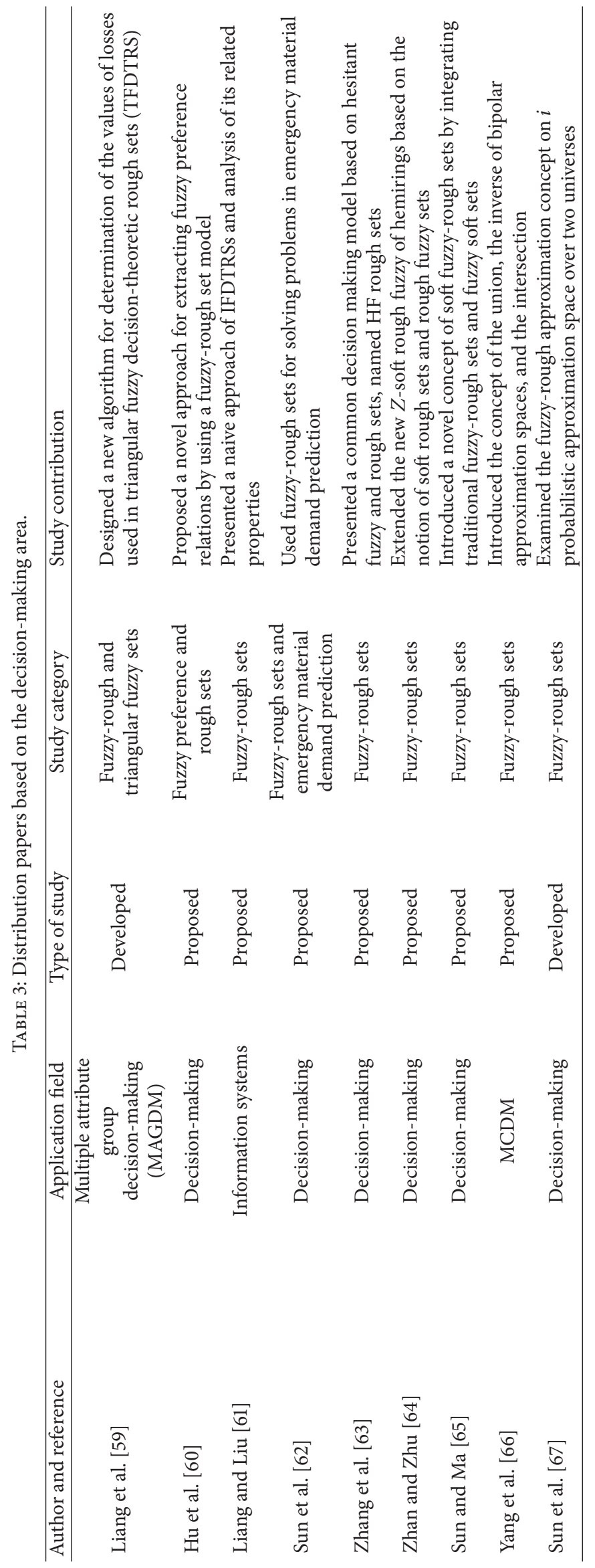


is the union of all cases that can be classified with certainty in one of the decision classes, whereas the upper approximation is a description of the instances that possibly belong to one of the decision classes. With the utilisation of rough set theory in the feature selection process, the vague concept can be modelled by the approximation of a vagueness set by a pair of precise concepts, called lower and upper approximations. The lower approximation, or positive region, is the union of all instances that can be classified with certainty in one of the decision values, whereas the upper approximation is a description of the instances that possibly belong to one of the decision values $[269,270]$. Some previous papers investigated some properties of fuzzy-rough approximation operators. $\mathrm{Hu}$ et al. [60] proposed a novel approach for extracting the fuzzy preference relations by using a fuzzy-rough set model. Zhang et al. [63] presented a common decision-making model based on hesitant fuzzy and rough sets, named HF rough sets. Sun and $\mathrm{Ma}$ [65] introduced a novel concept of soft fuzzy-rough sets by integrating the traditional fuzzy-rough sets and fuzzy soft sets. Table 4 provides valuable distribution findings of approximation operators based on the author and year of publication, application field, type of study, study category, and study contribution. The results represented in this table indicate that 15 articles have been published in the area of approximation operators.

5.4. Distribution Papers Based on Feature or Attribute Selection. There are several other instances of information that can be derived from data, but in this section we focus on classification. Formally, given a dataset of instances described by conditional features and a decision feature, classifiers aim to predict the class of a new instance given its conditional features. Most classifiers first build a model based on the data and then feed the new instance to the model to predict its class. For example, Support Vector Machines (SVMs) $[98,271-273]$ construct a function that models a separating border between the different classes in the data, and the value of that function for the new instance then determines to what class it most likely belongs. Decision trees [274, 275] generate rules from the data following a tree structure that predict the class of a new instance. Zhu et al. [276] introduced a novel attribute reduction criterion for choosing lowest attributes while keeping the best performance of the corresponding learning algorithms to some extent. Inbarani et al. [277] proposed a new feature selection approach based on high dimensionality in the medical dataset. This classifier has no modelling phase. A new instance is classified directly by looking up the closest instances in the data and classifying it to the class mostly occurring among those nearest neighbors. Before the data can be used to build the classification model and to classify the new instances, the data needs to be preprocessed. For example, there can be missing values [278-281] in the data that need to be imputed. Preprocessing can also be used to speed up or improve the classification process. For example, fuzzy-rough feature selection techniques select features such that the membership degrees of the instances to the fuzzy-rough positive region are maximised [195], or instance selection techniques select instances with a high membership degree to the fuzzy-rough positive region [195]. Jensen et al. [83] proposed a model by using a rough set for solving the problems related to the propositional satisfiability perspective. Qian et al. [84] proposed an approach based on dimensionality reduction together with sample reduction for a heuristic process of fuzzy-rough feature selection. Derrac et al. [88] presented a new hybrid algorithm for reduction of data using feature and instance selection. Jensen and Mac Parthaláin [89] introduced two novel, diverse ways of using the attribute and neighborhood approximation step for solving problems of complexity of the subset evaluation metric. Maji and Garai [91] presented a new feature selection approach based on fuzzy-rough sets by maximising the significance and relevance of the selected features. Zeng et al. [52] developed a new Hybrid Distance (HD) in Hybrid Information System (HIS) based on the value difference metric, and a new fuzzyrough approach by integrating the Gaussian kernel and HD distance. Cornelis et al. [95] introduced and extended a new rough set theory based on the multiadjoint fuzzy-rough sets for calculating the lower and upper approximations. Yao et al. [101] introduced a new fuzzy-rough approach called the variable precision $(\theta, \sigma)$ fuzzy-rough approach based on fuzzy granules. Zhao et al. [103] introduced a robust model for dimension reduction by using fuzzy-rough sets to achieve the possible parameters. Chen and Yang [104] integrated the rough set and fuzzy-rough set model for attribute reduction in decision systems with real and symbolic valued condition attributes. Table 5 provides valuable distribution findings of approximation operators based on the author and year of publication, application field, type of study, study category, and study contribution. The results represented in this table indicate that 23 articles have been published in the area of the attribute or attribute selection.

5.5. Distribution of Papers Based on Fuzzy Set Theories. In recent years, several studies have examined how rough set theory can be extended using various types of fuzzy set theories [53, 173, 238, 261, 282], which extends traditional set theory in the sense that instances can belong to a set to a certain degree between 0 and 1 . Most studies have been conducted on fuzzy sets with fuzzy-rough sets [36, $81,116,125,141,283,284]$, mainly focusing on preserving the predictive power of datasets with the least features possible. Some preliminary researches have been done on using fuzzy-rough set theory for instance selection [88] and its combination with fuzzy sets $[120,285]$. Apart from using fuzzy-rough set theory for preprocessing, it has also been used successfully to tackle classification problems directly, for example, in rule induction [157, 170, 286, 287], decisionmaking $[49,59,65,288]$ improving $K-\mathrm{NN}$ classification [87, 289-293], interval-valued fuzzy sets [108, 122, 130, 133, 294, 295], enhancing decision trees [165, 296, 297], hesitant fuzzy sets [132, 133, 137, 173, 298], and boosting SVMs [11, 98] [25, 26, 68, 106]. Huang et al. [106] proposed Dominance Intuitionistic Fuzzy Decision Tables (DIFDT) based on the fuzzy-rough set approach. Zhang [109] proposed intuitionistic fuzzy-rough sets based on intuitionistic fuzzy coverings by using intuitionistic fuzzy triangular norms and intuitionistic fuzzy implication operators. 


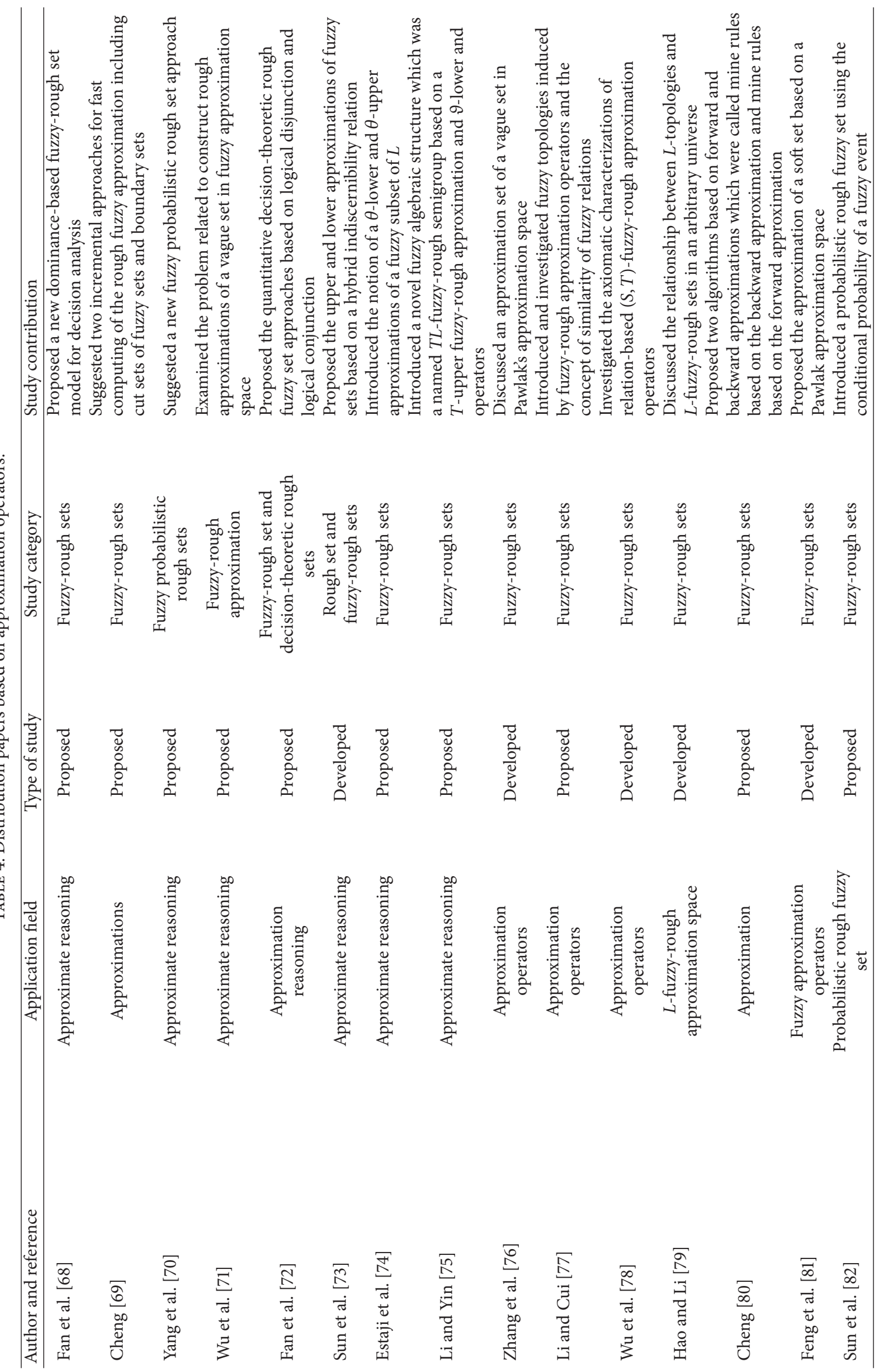




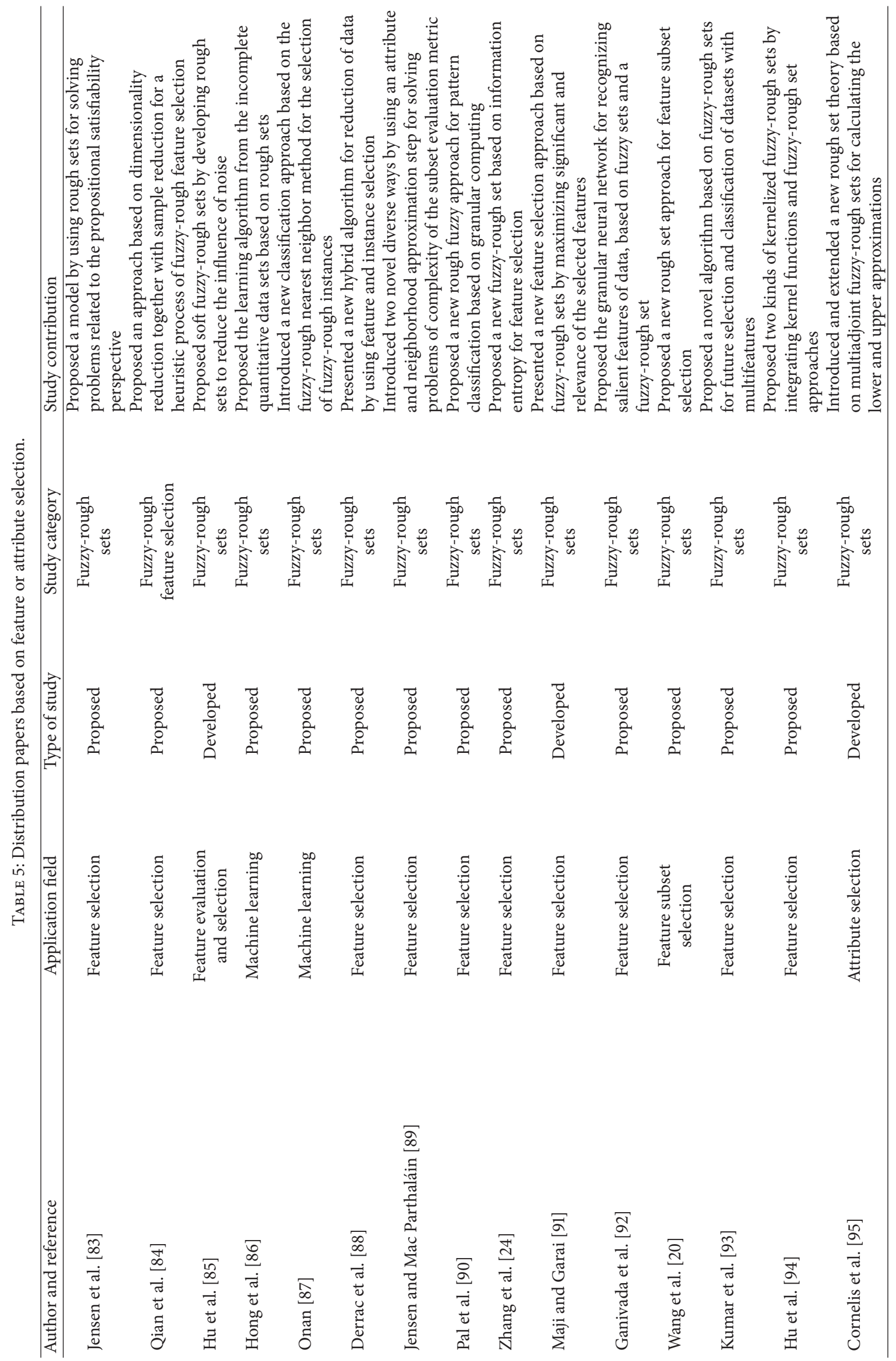




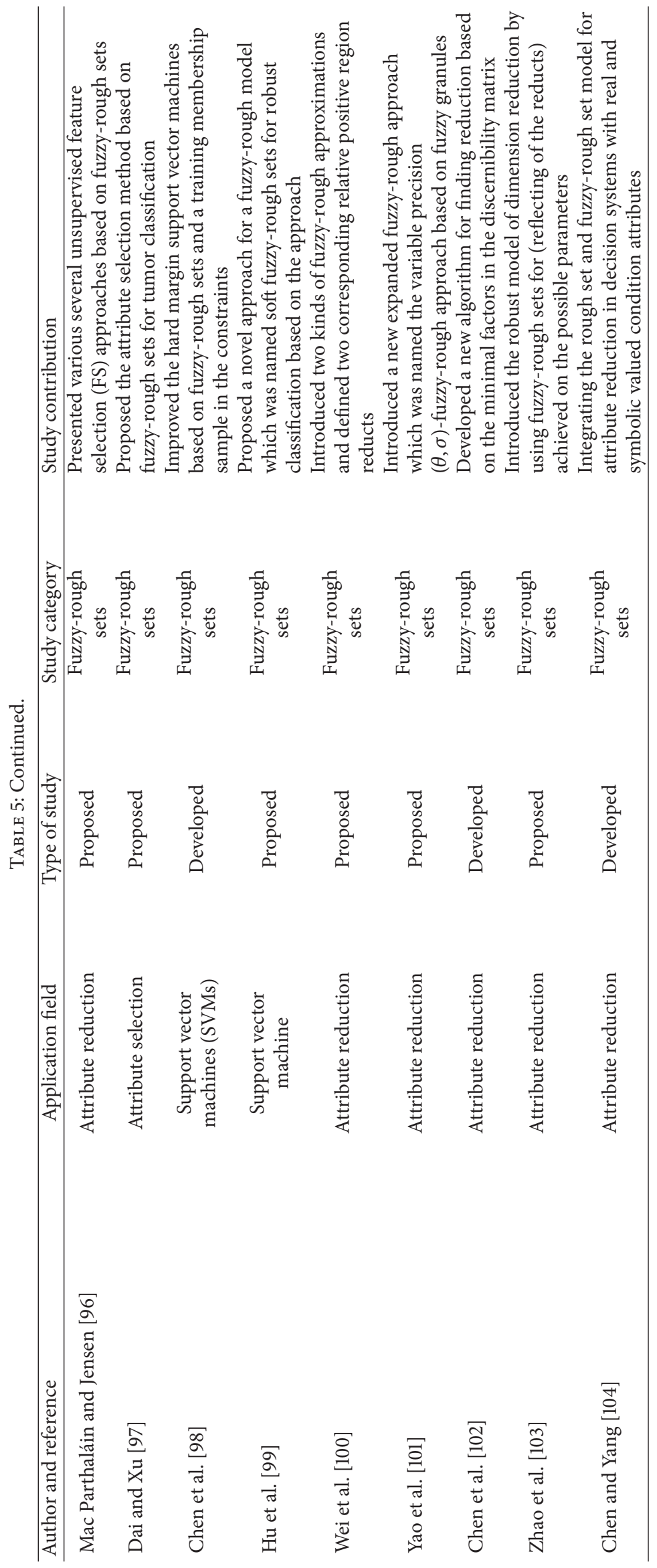


Zhang et al. [111] examined intuitionistic fuzzy-rough sets based on two universes, general binary relations, and an intuitionistic fuzzy implicator $I$ and a pair $(T, I)$ of the intuitionistic fuzzy $t$-norm $T$. Huang et al. [113] developed a novel multigranulation rough set which was named the Intuitionistic Fuzzy Multigranulation Rough Set (IFMGRS). Wang [114] examined type-2 fuzzy-rough sets based on extended $t$-norms and type 2 fuzzy relations in the convex normal fuzzy truth values. Chen et al. [117] introduced a geometrical interpretation and application of this type of membership functions. Ma [119] presented two novel types of fuzzy covering rough set for bridges linking covering rough sets and fuzzy-rough theory. Reference [29] investigated the topological characterizations of generalised fuzzy-rough regarding basic rough equalities. He et al. [120] proposed an inconsistent fuzzy decision system and reductions and improved discernibility matrix-based algorithms to discover reducts. Bai et al. [123] proposed an approach based on roughfuzzy sets for the extraction of spatial fuzzy decision rules from spatial data that simultaneously were of two kinds of fuzziness, roughness and uncertainties. Zhao and $\mathrm{Hu}$ [124] examined the fuzzy and interval-valued Fuzzy Probabilistic Rough Sets within frameworks of fuzzy and interval-valued fuzzy probabilistic approximation spaces. Huang et al. [125] introduced an Intuitionistic Fuzzy (IF) graded approximation space based on IF graded neighborhood and discussed information entropy and rough entropy measures. Li et al. [299] integrated the interval type 2 fuzzy with rough set theory by using the axiomatic and constructive approaches. Khuman et al. [126] investigated the type 2 fuzzy sets and rough-fuzzy sets to provide a practical means to express complex uncertainty without the associated difficulty of a type 2 fuzzy set. Zhang [295] introduced a new model based on interval-valued rough intuitionistic fuzzy sets by integrating the classical Pawlak rough set and interval-valued IF set theory. $\mathrm{Hu}$ [130] developed an integrative model considering interval-valued fuzzy sets and variable precision named generalised interval-valued fuzzy variable precision rough sets. Yang et al. [132] investigated a novel fuzzy-rough set model based on constructive and axiomatic approaches to introduce the hesitant fuzzy-rough set model. Zhang et al. [133] integrated the interval-valued hesitant fuzzy sets with rough sets to introduce the novel model named the intervalvalued hesitant fuzzy-rough set. Tiwari and Srivastava [300] investigated the results of some previous studies regarding the one-to-one correspondence between the family of fuzzy preorders on a nonempty set. Chen et al. [136] proposed a new rough-fuzzy approach for handling, representation, and utilisation of various levels of uncertainty in knowledge. Table 6 provides valuable distribution results of fuzzy sets theories based on the author and year of publication, application field, type of study, study category, and study contribution. The results represented in this table indicate that 37 articles have been published in the area of the attribute or attribute selection.

5.6. Distribution of Papers Based on Other Application Areas. In recent decades, rough sets and fuzzy-rough sets theories have been employed in various application areas such as data mining [4, 5, 86, 301-303], software packages [141, $304]$, web ontology [138, 305-307], pattern recognition [24, $148,187,308-310]$, granular computing [38, 221, 238, 251], genetic algorithm [310-313], prototype selection [145, 163], solid transportation [146, 314, 315], social networks [316$318]$, artificial neural network [92, 153, 319], remote sensing $[320,321]$, and gene selection $[158,322-324]$. An et al. [140] analysed a regression algorithm based on fuzzy partition, fuzzy-rough sets, estimation of regression values, and fuzzy approximation for estimating wind speed. Shiraz et al. [142] proposed a new fuzzy-rough DEA approach by combining the classical DEA, rough set, and fuzzy set theory to accommodate the uncertainty. Zhou et al. [144] developed a new approach for automatic selection of the threshold parameter to determine the approximation regions in rough set-based clustering. Vluymans et al. [19] introduced a novel kind of classifier for imbalanced multi-instance data based on fuzzy-rough set theory. Ganivada et al. [150] proposed a Fuzzy-Rough Granular Self-Organising Map (FRGSOM) by including the threedimensional linguistic vector and connection weights for clustering the patterns which included overlapping regions. Amiri and Jensen [151] introduced three missing imputation approaches based on the fuzzy-rough nearest neighbors, namely, VQNNI, OWANNI, and FRNNI. Feng and Mi [39] introduced the use of data mining approaches to forecast the need of maintenance. Affonso et al. [153] proposed a new method for biological image classification by a roughfuzzy artificial neural network. Pahlavani et al. [155] proposed a novel fuzzy-rough set model to extract the rules in the ANFIS based classification procedure for choosing the optimum features. Zhao et al. [157] developed a rulebased classifier fuzzy-rough using one generalised fuzzyrough set model to introduce a novel idea which was called consistence degree. Maji and Pal [158] presented a new fuzzy equivalence partition matrix for approximating the true marginal and joint distributions of continuous gene expression values. Huang and Kuo [159] investigated two perspectives of cross-lingual semantic document similarity measures based on the fuzzy sets and rough sets which were named formulation of similarity measures and document representation. Ramentol et al. [161] developed a learning algorithm for considering the imbalance representation and proposed a classification algorithm for imbalanced data by using the fuzzy-rough sets and ordered weighted average aggregation. Derrac et al. [163] introduced a new fuzzyrough set model for prototype selection by optimising the behaviour of this classifier. Zhao et al. [166] introduced a novel complement information entropy method in the fuzzyrough sets based on the arbitrary fuzzy relations, inner-class, and outer-class information. Changdar et al. [168] presented a new genetic-ant colony optimisation algorithm in a fuzzyrough set environment for solving problems related to the solid multiple Travelling Salesmen Problem (mTSP). Sun and $\mathrm{Ma}$ [169] introduced a novel model to evaluate the emergency plants for unconventional emergency events using soft fuzzyrough set theory. The results of this section are provided in Table 7. 


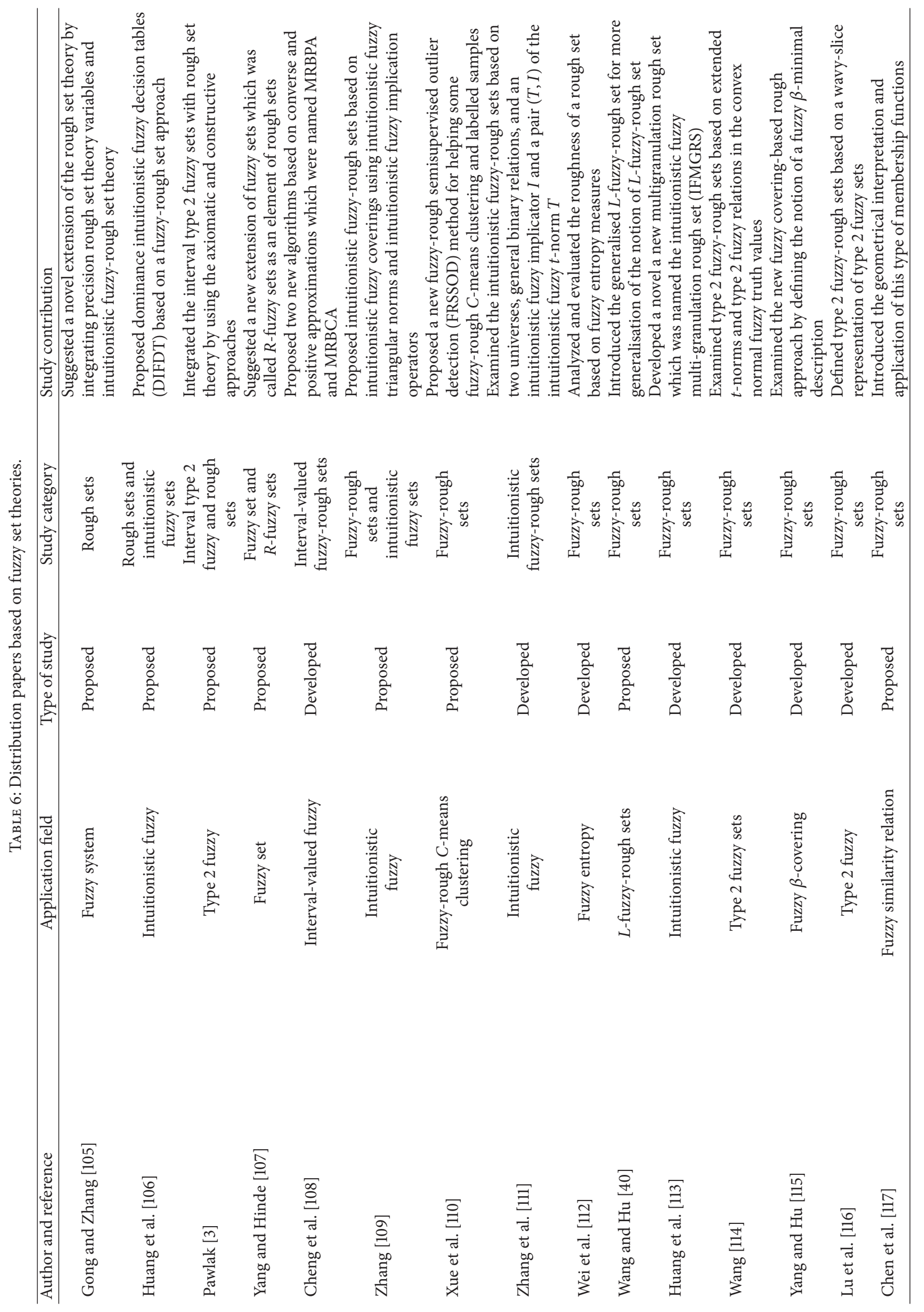




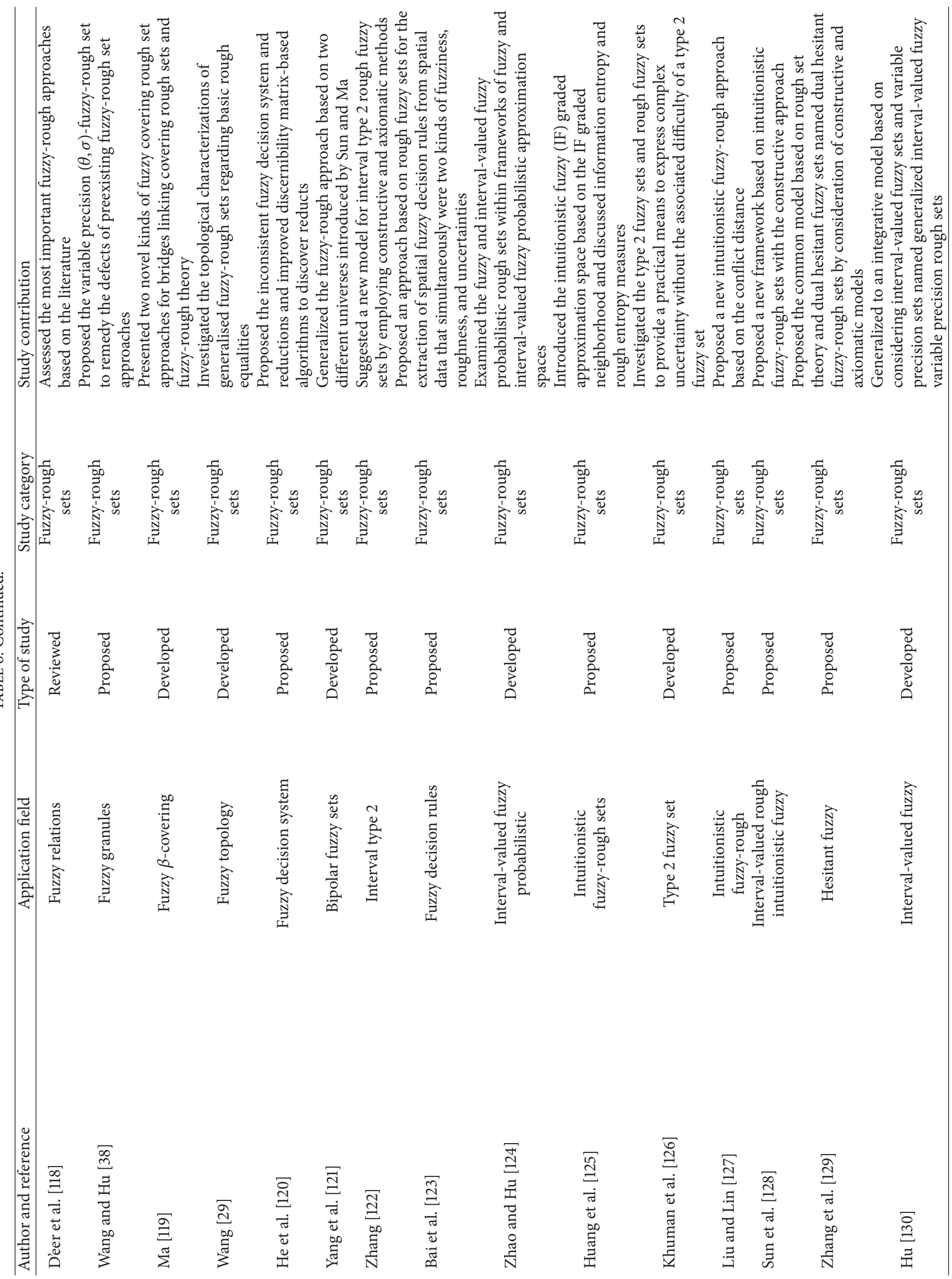




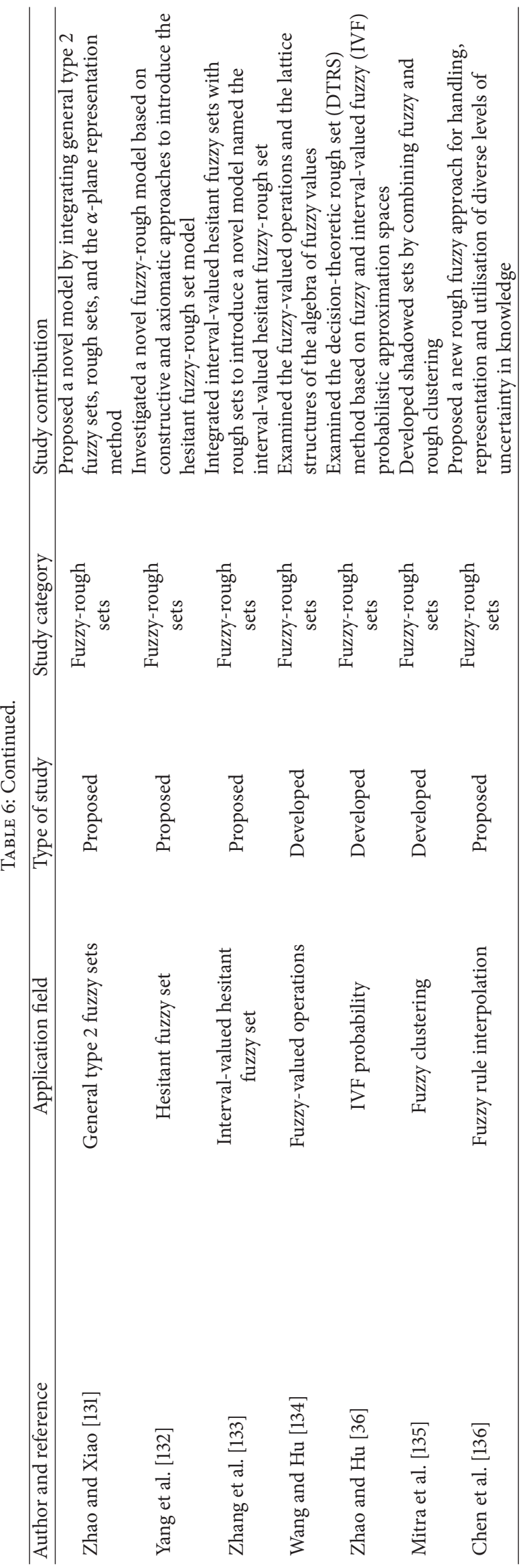




\section{Distribution of Reviewed Paper by Journals}

Table 8 presents the results of analysing the articles based on distribution of the journals. The articles related to the fuzzyrough set theory have been chosen from 28 different international scholarly journals indexed in the Web of Science databases. Selected articles published, along with an extensive diversity of journals that focus on fuzzy-rough set theory, validate different scholarly journals willingness to publish in this field. By far, the highest ranking journal is the journal of Information Sciences with 36 articles, followed by the journal of Fuzzy sets and Systems with 17 papers. Furthermore, more than 60 percent of the total papers (83 out of 132 papers) were concentrated in five journals, which play dominant roles in field of rough-fuzzy set theory. Additionally, in other rankings, the Soft Computing journal had the third rank with 11 publications followed by journal of Knowledge-Based Systems and IEEE Transactions on Fuzzy Systems with 10 articles. Hence, based on this result, we can conclude that these selected journals can be considered as the main journals on the fuzzy-rough set theory, as the more than 60 percent of the articles were published in these journals. Table 8 presents the list of journals where the fuzzy-rough set theory has been published.

\section{Distribution of Articles by Year of Publication}

In recent decades, the application of fuzzy-rough set theory has increased dramatically in the literature. A historical growth of fuzzy-rough sets has existed for many years. A frequency analysis of the 132 articles based on the articles published for different years is shown in Figure 4. During 2010-2012, the articles published on fuzzy-rough set theory were at a steady rate with 15,11 , and 15 articles. The uptrend in papers outputs is observed since the year 2012, until 2016. Figure 4 presents relevant information based on the frequency of distribution by the year of publication. Accordingly, it can be indicated that nowadays researchers are highly interested in conducting research on fuzzy-rough set theory and it can be predicted that in coming years these numbers will increase.

\section{Distribution of Papers Based on Nationality of Authors}

This review paper attempted to show the difference among the countries related to the fuzzy-rough set theory. Two kinds of principles were used for identifying the characteristics in selected articles, including the information gained directly from the papers or the nationality of the first author. Figure 5 shows that authors from 16 nationalities and countries investigated the fuzzy-rough set theory. Most of the published papers were from China with 86 publications followed by India, United Kingdom, and Spain with 13, 6, and 5 publications, respectively. Figure 5 shows the frequency of other nationalities, as well.

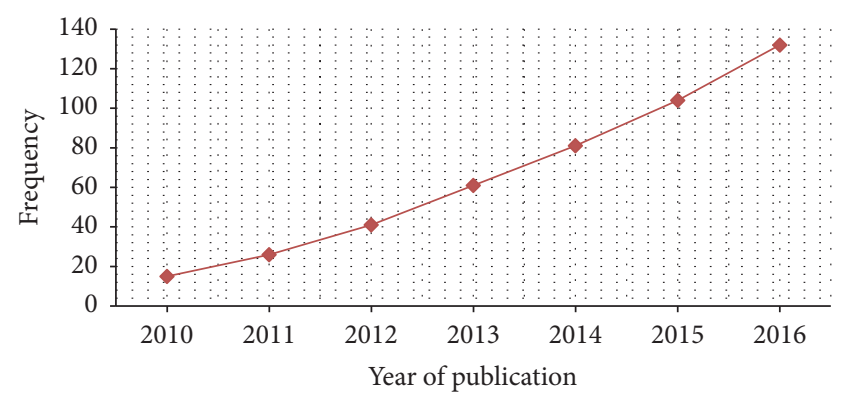

FIGURE 4: Distribution of articles by year of publication.

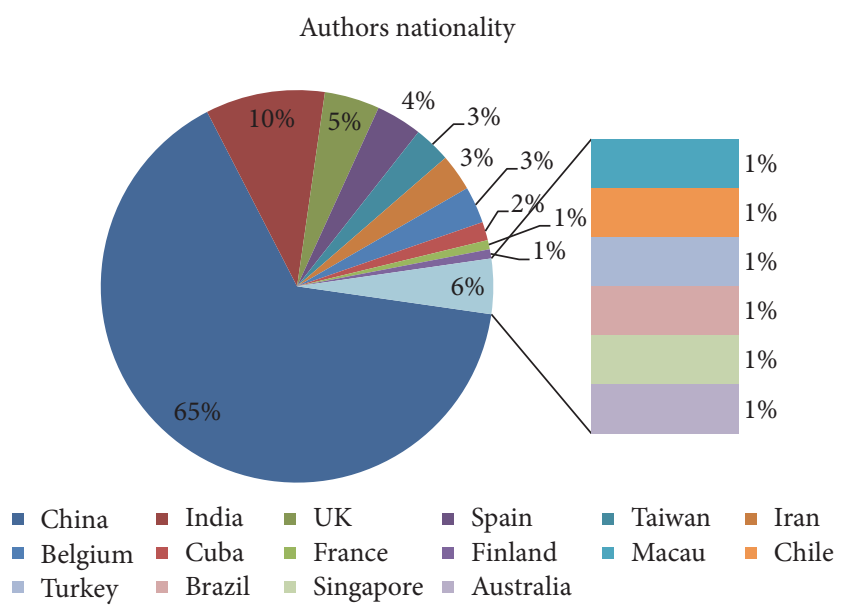

FIGURE 5: Distribution of papers based on nationality of authors.

\section{Discussion}

There are some challenges regarding various application areas of fuzzy-rough set theory that can be interesting for discussion and future studies. For example, in the case of fuzzy $\beta$-covering approximation spaces, there are various topics which need further research, such as matroidal structures, data mining, the generalisation of fuzzy covering-based rough sets, topological properties and data compression with homomorphism, and communication by using fuzzy covering-based rough sets. In the area of multigranulation fuzzy-rough set theory, some more investigations are needed. For example, it is necessary to explore the primary theory and characterizations of multigranulation fuzzy-rough sets over two universes, as well as attribute reduction of the multigranulation fuzzy approximation space over two universes. In the field of fuzzy information system over Two Universes (ISTU), further study is necessary to enhance the current incremental algorithms by integration with the parallelism technique to update rough approximations. More investigations are required in the area of interval-valued hesitant fuzzy multigranulation rough sets over two universes, to study uncertainty measures, topological structures, and attribute reduction methods. Also, further investigations are needed in the area of the Dominance-Based Fuzzy-Rough Set Approach (DFRSA), by improving the attribute reduction, rule induction, and object reduction. In the field of fuzzy 


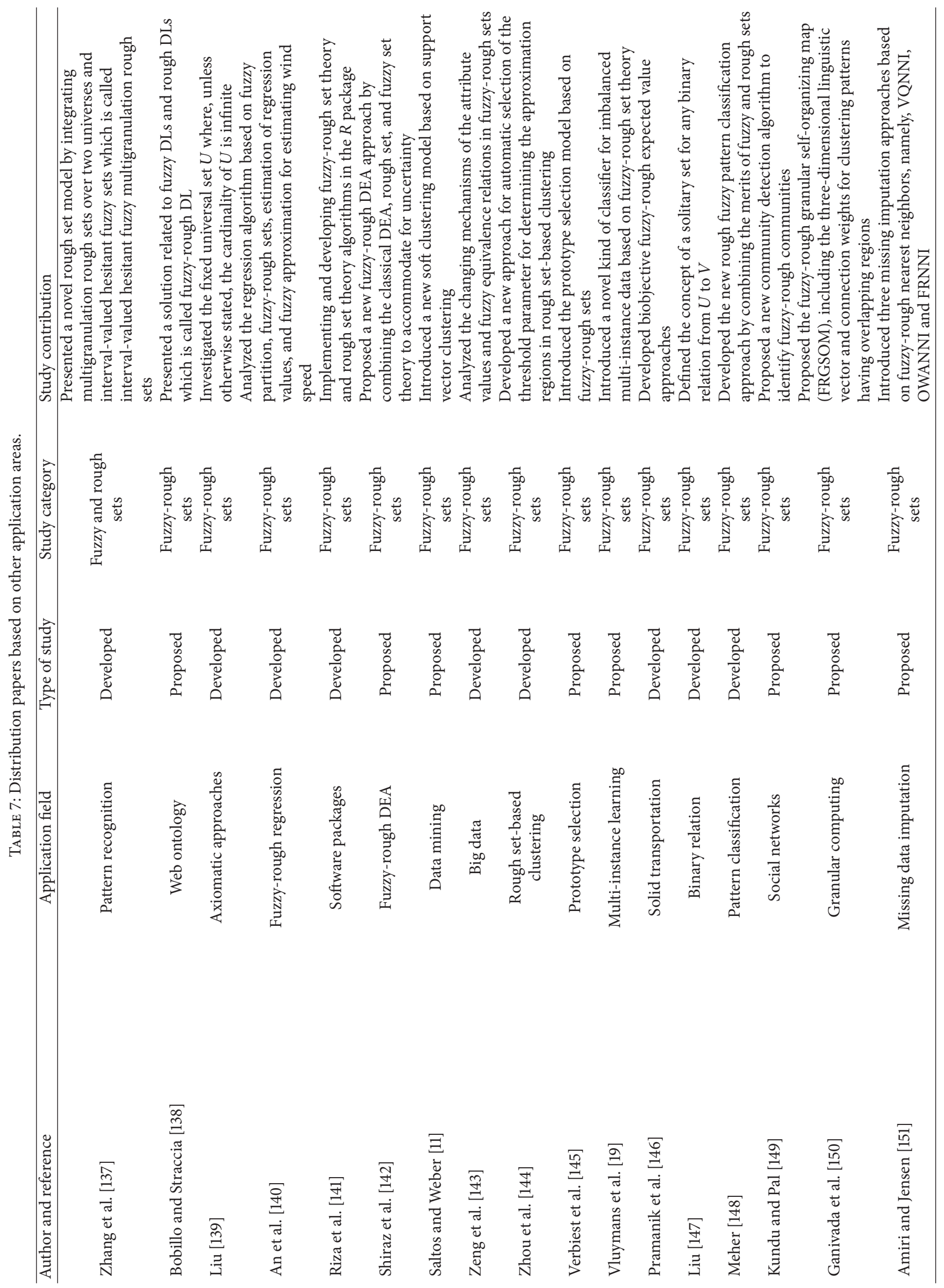




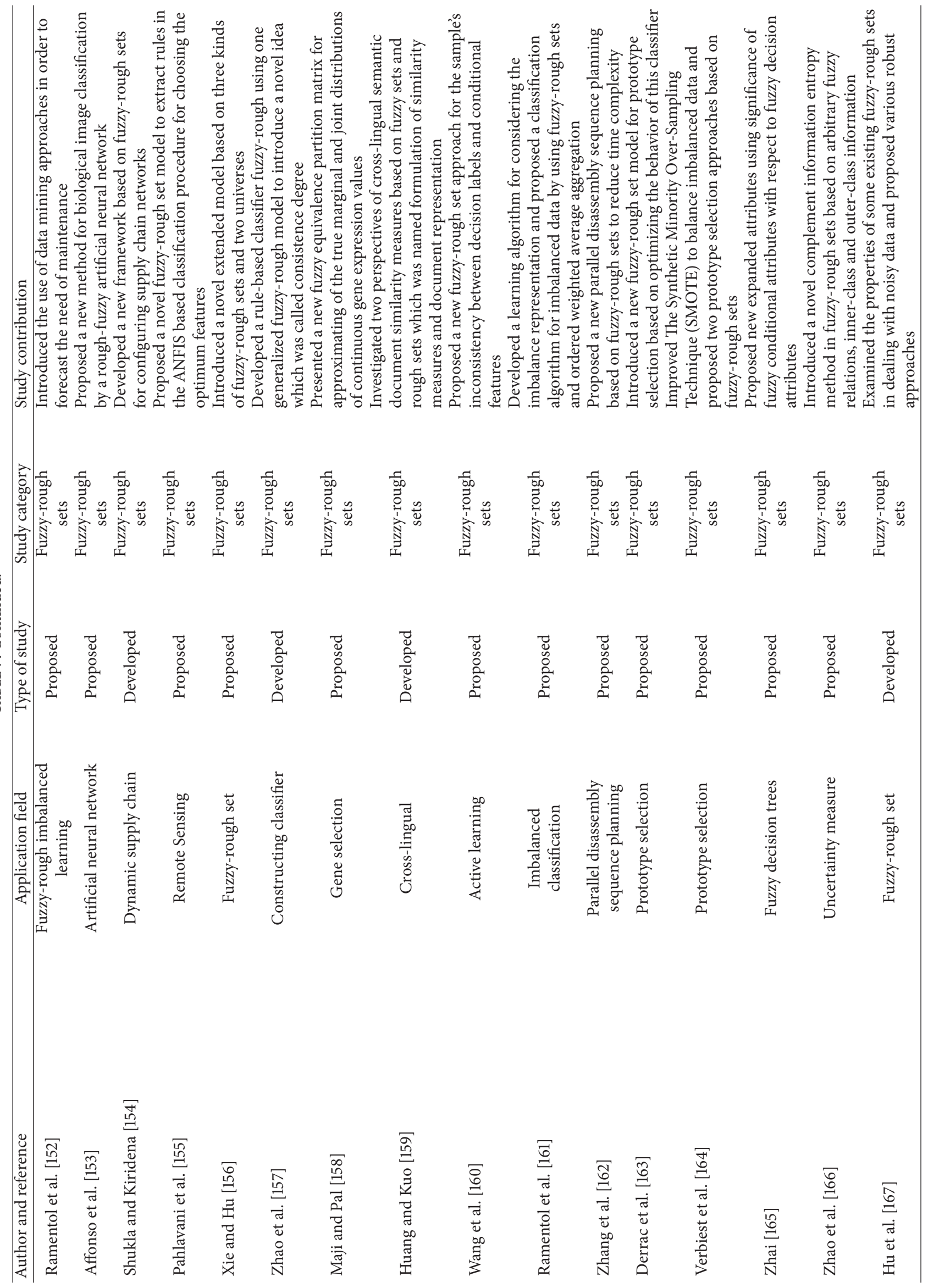




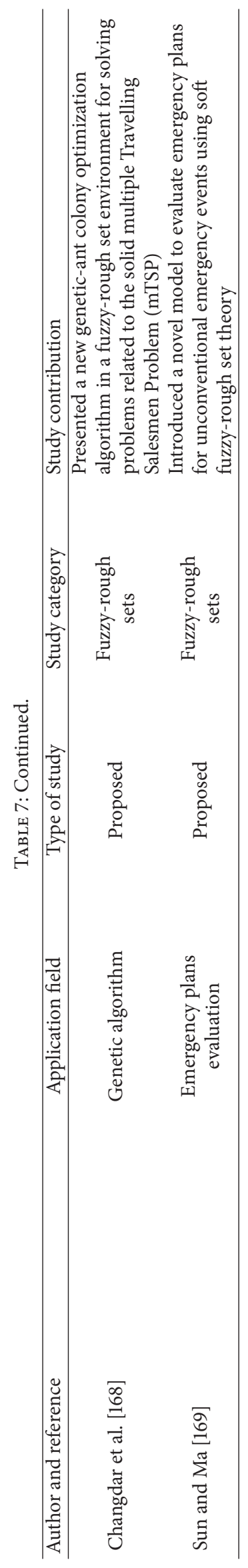


TABLE 8: Distribution of papers based on the name of journals.

\begin{tabular}{|c|c|c|}
\hline Name of journals & Frequently & Percentage \\
\hline Information Sciences & 36 & $27.27 \%$ \\
\hline Fuzzy sets and Systems & 15 & $11.36 \%$ \\
\hline Soft Computing & 11 & $8.33 \%$ \\
\hline Knowledge-Based Systems & 10 & $7.58 \%$ \\
\hline IEEE Transactions On Fuzzy Systems & 10 & $7.58 \%$ \\
\hline Pattern Recognition & 6 & $4.55 \%$ \\
\hline International Journal of General Systems & 5 & $3.79 \%$ \\
\hline Journal of Approximate Reasoning & 5 & $3.79 \%$ \\
\hline Expert Systems with Applications & 5 & $3.79 \%$ \\
\hline Applied Soft Computing & 5 & $3.79 \%$ \\
\hline Applied Mathematical Modelling & 4 & $3.03 \%$ \\
\hline Pattern Recognition Letters & 2 & $1.52 \%$ \\
\hline IEEE Transactions on Knowledge and Data Engineering & 2 & $1.52 \%$ \\
\hline Neurocomputing & 2 & $1.52 \%$ \\
\hline Artificial Intelligence Review & 1 & $0.76 \%$ \\
\hline Expert Systems & 1 & $0.76 \%$ \\
\hline Data \& Knowledge Engineering & 1 & $0.76 \%$ \\
\hline Neural Networks & 1 & $0.76 \%$ \\
\hline Mathematics and Computers in Simulation & 1 & $0.76 \%$ \\
\hline Fundamenta Informaticae & 1 & $0.76 \%$ \\
\hline Socio-Economic Planning Sciences & 1 & $0.76 \%$ \\
\hline Theoretical Computer Science & 1 & $0.76 \%$ \\
\hline Engineering Applications of Artificial Intelligence & 1 & $0.76 \%$ \\
\hline International Journal of Production Research & 1 & $0.76 \%$ \\
\hline International Journal of Remote Sensing & 1 & $0.76 \%$ \\
\hline The International Journal of Advanced Manufacturing Technology & 1 & $0.76 \%$ \\
\hline Kybernetes & 1 & $0.76 \%$ \\
\hline Mathematics and Computers in Simulation & 1 & $0.76 \%$ \\
\hline Total & 132 & $100.00 \%$ \\
\hline
\end{tabular}

neighborhood rough sets, further study is necessary regarding classification learning and reasoning with uncertainty. In addition, in the area of type 2 fuzzy-rough sets, further investigations are required regarding attribute reduction related to granular type 2 fuzzy sets and various methods of knowledge discovery in the complex fuzzy information systems. Furthermore, although some studies have investigated type-2 variable precision multigranulation fuzzy-rough sets, future studies can focus on uncertainty and reduction measures of variable precision multigranulation fuzzy-rough sets. Moreover, although some scholars have examined Fuzzy Probabilistic Rough Sets (FPRSs) and Interval-Valued Fuzzy Probabilistic Rough Sets (IVF-PRSs) with models of IVF and fuzzy probabilistic approximation spaces, there is still need to focus on characterizations of axiomatic methods in FPRSs and IVF-PRSs models. Although some previous papers investigated Gaussian Kernel Fuzzy-Rough Sets (FRS) in Hybrid Information Systems (HIS) further studies are required regarding technologies of parallel computing, for example, Map Reduce to optimise the increment updating model. There are also some papers regarding intuitionistic fuzzy-rough set model. However, more investigation is necessary to improve and construct the dominance intuitionistic fuzzy variable precision rough sets theory models. Although some previous papers examined the DTRS approach in the area of fuzzy and IVF probabilistic approximation spaces, there is a need for more studies to be conducted on the circumstance of IVF sets. In addition, there are some works related to IFDTRSs focusing on decision-theoretic rough sets; nonetheless, there is a need for more investigations on generalisation models based on the IFDTRSs. Although some previous researchers focused on the fuzzy topologies induced by the fuzzy-rough approximation operators, further articles are necessary for enhancing the generalised similarity of fuzzy relations, based on the $t$-norms and complete residuated lattices. Furthermore, although papers were published regarding the Intuitionistic Fuzzy Multigranulation Rough Sets (IFMGRS), further enhancements of these models are required by focusing on an interval-valued IF environment.

\section{Conclusion}

This review paper presented a comprehensive overview of recent fuzzy generations of rough sets theory in various applications areas. In total, 132 papers were selected for systematic review and meta-analysis in the period 2010-2016 
from popular international journals accessible in the Web of Science database. We carefully selected and reviewed 132 studies about the fuzzy-rough set theory based on the title, abstract, introduction, research methods, and conclusions. These selected papers were categorised into six application areas. Also, all papers were classified based on the author and year of publication, author nationalities, application field, type of study, study category, study contribution, and journal in which they appeared. Some points on fuzzy-rough set theory were gained from this review article. The vast majority of reviewed articles were published between 2013 and 2016. In total, the papers were classified into six areas including information systems, decision-making, approximation operators, feature and attribute selection, fuzzy set theories, and other application areas. Fuzzy set theory combined with rough set theory was the most important application area with 37 papers. Furthermore, 28 international peer reviewed journals were considered in the current review paper. The Journal of Information Systems had the first rank among the considered journals regarding publishing papers related to the fuzzy-rough set theory. The articles published at the beginning of 2017 (if any) have not been included in the present paper because of the limited reporting time. We attempted to use those published articles in other sections of our review paper, such as related works and introduction sections. However, this present review can be developed for the future studies. Another limitation is that the data was collected from journals, while the examined documents did not include textbooks, doctoral and master's theses, and unpublished papers on MCDM problems. Although we attempted to provide the comprehensive review based on the current and old literature, nevertheless, as a recommendation for future studies, the data can be collected from these sources, and the obtained results can be compared with the data obtained and reported in this study. Another limitation of this review was that all of the papers were extracted from the journals written in English. Hence, scientific journals in other languages were not included in the review. However, the researchers believe that this paper comprehensively reviewed most of the papers published in international journals. In this paper, we reviewed 132 papers which recently studied generalised fuzzy-rough sets theory but attempted to include the comprehensive list of papers in other sections. In addition, we carefully selected and summarised the available papers of several publishers in the Web of Science database. However, some relevant outlets remained beyond the scope of the current study. Therefore, future researchers will be able to review those papers which are not considered in the current review. Another limitation of the survey is that although the paper presents various journals and conference publications that recently studied generalised fuzzy-rough set models, it does not include any of this topic discussed in the published books.

\section{Conflicts of Interest}

The authors declare no conflicts of interest regarding the publication of the paper.

\section{References}

[1] C.-C. Yeh, D.-J. Chi, and M.-F. Hsu, "A hybrid approach of DEA, rough set and support vector machines for business failure prediction," Expert Systems with Applications, vol. 37, no. 2, pp. 1535-1541, 2010.

[2] F. E. H. Tay and L. Shen, "Economic and financial prediction using rough sets model," European Journal of Operational Research, vol. 141, no. 3, pp. 641-659, 2002.

[3] Z. a. Pawlak, "Rough sets," International Journal of Computer and Information Sciences, vol. 11, no. 5, pp. 341-356, 1982.

[4] J. Zhang, T. Li, and H. Chen, "Composite rough sets for dynamic data mining," Information Sciences. An International Journal, vol. 257, pp. 81-100, 2014.

[5] Y. Huang, T. Li, C. Luo, H. Fujita, and S.-J. Horng, "Matrixbased dynamic updating rough fuzzy approximations for data mining," Knowledge-Based Systems, vol. 119, pp. 273-283, 2017.

[6] J. Hu, T. Li, C. Luo, H. Fujita, and Y. Yang, "Incremental fuzzy cluster ensemble learning based on rough set theory," Knowledge-Based Systems, vol. 132, pp. 144-155, 2017.

[7] M. Ye, X. Wu, X. Hu, and D. Hu, "Anonymizing classification data using rough set theory," Knowledge-Based Systems, vol. 43, pp. 82-94, 2013.

[8] I.-K. Park and G.-S. Choi, "Rough set approach for clustering categorical data using information-theoretic dependency measure," Information Systems, vol. 48, pp. 289-295, 2015.

[9] F. Shi, S. Sun, and J. Xu, "Employing rough sets and association rule mining in KANSEI knowledge extraction," Information Sciences, vol. 196, pp. 118-128, 2012.

[10] F. Pacheco, M. Cerrada, R.-V. Sánchez, D. Cabrera, C. Li, and J. Valente de Oliveira, "Attribute clustering using rough set theory for feature selection in fault severity classification of rotating machinery," Expert Systems with Applications, vol. 71, pp. 69-86, 2017.

[11] R. Saltos and R. Weber, "A Rough-Fuzzy approach for Support Vector Clustering," Information Sciences, vol. 339, pp. 353-368, 2016.

[12] D. Chen, Y. Yang, and Z. Dong, "An incremental algorithm for attribute reduction with variable precision rough sets," Applied Soft Computing Journal, vol. 45, pp. 129-149, 2016.

[13] E.-S. M. El-Alfy and M. A. Alshammari, "Towards scalable rough set based attribute subset selection for intrusion detection using parallel genetic algorithm in MapReduce," Simulation Modelling Practice and Theory, vol. 64, pp. 18-29, 2015.

[14] S. Eskandari and M. M. Javidi, "Online streaming feature selection using rough sets," International Journal of Approximate Reasoning, vol. 69, pp. 35-57, 2016.

[15] J. G. and H. Inbarani H., "Hybrid Tolerance Rough Set-Firefly based supervised feature selection for MRI brain tumor image classification," Applied Soft Computing Journal, vol. 46, pp. 639651, 2016.

[16] C. Hu, S. Liu, and X. Huang, "Dynamic updating approximations in multigranulation rough sets while refining or coarsening attribute values," Knowledge-Based Systems, vol. 130, pp. 62-73, 2017.

[17] H. Li, D. Li, Y. Zhai, S. Wang, and J. Zhang, "A novel attribute reduction approach for multi-label data based on rough set theory," Information Sciences, vol. 367-368, pp. 827-847, 2016.

[18] M. S. Raza and U. Qamar, "An incremental dependency calculation technique for feature selection using rough sets," Information Sciences. An International Journal, vol. 343/344, pp. 41-65, 2016. 
[19] S. Vluymans, D. Sánchez Tarragó, Y. Saeys, C. Cornelis, and F. Herrera, "Fuzzy rough classifiers for class imbalanced multiinstance data," Pattern Recognition, vol. 53, pp. 36-45, 2016.

[20] C. Wang, M. Shao, Q. He, Y. Qian, and Y. Qi, "Feature subset selection based on fuzzy neighborhood rough sets," KnowledgeBased Systems, vol. 111, pp. 173-179, 2016.

[21] Y. Yao and X. Zhang, "Class-specific attribute reducts in rough set theory," Information Sciences. An International Journal, vol. 418/419, pp. 601-618, 2017.

[22] J. Zhai, X. Wang, and X. Pang, "Voting-based instance selection from large data sets with MapReduce and random weight networks," Information Sciences, vol. 367-368, pp. 1066-1077, 2016.

[23] H.-Y. Zhang and S.-Y. Yang, "Feature selection and approximate reasoning of large-scale set-valued decision tables based on $\alpha$-dominance-based quantitative rough sets," Information Sciences, vol. 378, pp. 328-347, 2017.

[24] X. Zhang, C. Mei, D. Chen, and J. Li, "Feature selection in mixed data: a method using a novel fuzzy rough set-based information entropy," Pattern Recognition, pp. 1-15, 2016.

[25] C. Wang, Y. Qi, M. Shao et al., "A Fitting Model for Feature Selection With Fuzzy Rough Sets," IEEE Transactions on Fuzzy Systems, vol. 25, no. 4, pp. 741-753, 2017.

[26] A. Sanchis, M. J. Segovia, J. A. Gil, A. Heras, and J. L. Vilar, "Rough Sets and the role of the monetary policy in financial stability (macroeconomic problem) and the prediction of insolvency in insurance sector (microeconomic problem)," European Journal of Operational Research, vol. 181, no. 3, pp. 1554-1573, 2007.

[27] P. Pattaraintakorn, N. Cercone, and K. Naruedomkul, "Rule learning: ordinal prediction based on rough sets and softcomputing," Applied Mathematics Letters. An International Journal of Rapid Publication, vol. 19, no. 12, pp. 1300-1307, 2006.

[28] A. M. Radzikowska and E. E. Kerre, "A comparative study of fuzzy rough sets," Fuzzy Sets and Systems, vol. 126, no. 2, pp. 137$155,2002$.

[29] C. Y. Wang, “Topological characterizations of generalized fuzzy rough sets," Fuzzy Sets and Systems, vol. 312, pp. 109-125, 2017.

[30] C. Y. Wang, "Topological structures of L-fuzzy rough sets and similarity sets of L-fuzzy relations," International Journal of Approximate Reasoning, vol. 83, pp. 160-175, 2017.

[31] W. Pan, K. She, and P. Wei, "Multi-granulation fuzzy preference relation rough set for ordinal decision system," Fuzzy Sets and Systems. An International Journal in Information Science and Engineering, vol. 312, pp. 87-108, 2017.

[32] A. Namburu, S. K. Samay, and S. R. Edara, "Soft fuzzy rough setbased MR brain image segmentation," Applied Soft Computing Journal, vol. 54, pp. 456-466, 2017.

[33] Y. Li, S. Wu, Y. Lin, and J. Liu, "Different classes' ratio fuzzy rough set based robust feature selection," Knowledge-Based Systems, vol. 120, pp. 74-86, 2017.

[34] T. Feng, H.-T. Fan, and J.-S. Mi, "Uncertainty and reduction of variable precision multigranulation fuzzy rough sets based on three-way decisions," International Journal of Approximate Reasoning, vol. 85, pp. 36-58, 2017.

[35] B. Sun, W. Ma, and Y. Qian, "Multigranulation fuzzy rough set over two universes and its application to decision making," Knowledge-Based Systems, vol. 123, pp. 61-74, 2017.

[36] X. R. Zhao and B. Q. Hu, "Fuzzy and interval-valued fuzzy decision-theoretic rough set approaches based on fuzzy probability measure," Information Sciences. An International Journal, vol. 298, pp. 534-554, 2015.
[37] H. Zhang and L. Shu, "Generalized interval-valued fuzzy rough set and its application in decision making," International Journal of Fuzzy Systems, vol. 17, no. 2, pp. 279-291, 2015.

[38] C. Y. Wang and B. Q. Hu, "Granular variable precision fuzzy rough sets with general fuzzy relations," Fuzzy Sets and Systems. An International Journal in Information Science and Engineering, vol. 275, pp. 39-57, 2015.

[39] T. Feng and J.-S. Mi, "Variable precision multigranulation decision-theoretic fuzzy rough sets," Knowledge-Based Systems, vol. 91, pp. 93-101, 2016.

[40] C. Y. Wang and B. Q. Hu, "Fuzzy rough sets based on generalized residuated lattices," Information Sciences. An International Journal, vol. 248, pp. 31-49, 2013.

[41] S. Teng, F. Liao, M. He, M. Lu, and Y. Nian, "Integrated measures for rough sets based on general binary relations," SpringerPlus, vol. 5, no. 1, article no. 117, pp. 1-17, 2016.

[42] F. Maciá-Pérez, J. V. Berna-Martinez, A. Fernández Oliva, and M. A. Abreu Ortega, "Algorithm for the detection of outliers based on the theory of rough sets," Decision Support Systems, vol. 75, Article ID 12609, pp. 63-75, 2015.

[43] J. J. Buckley and E. Eslami, An Introduction to Fuzzy Logic and Fuzzy Sets, Springer Science and Business Media, 2002.

[44] L. A. Zadeh, "Fuzzy sets," Information and Control, vol. 8, no. 3, pp. 338-353, 1965.

[45] B. Bede and S. G. Gal, "Almost periodic fuzzy-number-valued functions," Fuzzy Sets and Systems. An International Journal in Information Science and Engineering, vol. 147, no. 3, pp. 385-403, 2004.

[46] Z. Pawlak, "Rough set theory and its applications to data analysis," Cybernetics and Systems, vol. 29, no. 7, pp. 661-688, 1998.

[47] L. Polkowski, S. Tsumoto, and T. Y. Lin, Rough Set Methods and Applications: New Developments in Knowledge Discovery in Information Systems, Physica-Verlag GmbH, Heidelberg, Germany, 2000.

[48] G. Liu and Y. Sai, "Invertible approximation operators of generalized rough sets and fuzzy rough sets," Information Sciences. An International Journal, vol. 180, no. 11, pp. 2221-2229, 2010.

[49] Z. Zhang, "A rough set approach to intuitionistic fuzzy soft set based decision making," Applied Mathematical Modelling. Simulation and Computation for Engineering and Environmental Systems, vol. 36, no. 10, pp. 4605-4633, 2012.

[50] Y. Ouyang, Z. Wang, and H.-p. Zhang, "On fuzzy rough sets based on tolerance relations," Information Sciences. An International Journal, vol. 180, no. 4, pp. 532-542, 2010.

[51] J. Dai and H. Tian, "Fuzzy rough set model for set-valued data," Fuzzy Sets and Systems. An International Journal in Information Science and Engineering, vol. 229, pp. 54-68, 2013.

[52] A. Zeng, T. Li, D. Liu, J. Zhang, and H. Chen, "A fuzzy rough set approach for incremental feature selection on hybrid information systems," Fuzzy Sets and Systems. An International Journal in Information Science and Engineering, vol. 258, pp. 39$60,2015$.

[53] M. Shabir and T. Shaheen, "A new methodology for fuzzification of rough sets based on $\alpha$-indiscernibility," Fuzzy Sets and Systems. An International Journal in Information Science and Engineering, vol. 312, pp. 1-16, 2017.

[54] X. Wu and J.-L. Zhang, "Rough set models based on random fuzzy sets and belief function of fuzzy sets," International Journal of General Systems, vol. 41, no. 2, pp. 123-141, 2012. 
[55] S.-M. Chen, S.-H. Cheng, and Z.-J. Chen, "Fuzzy interpolative reasoning based on the ratio of fuzziness of rough-fuzzy sets," Information Sciences. An International Journal, vol. 299, pp. 394-411, 2015.

[56] E. C. C. Tsang, C. Wang, D. Chen, C. Wu, and Q. Hu, "Communication between information systems using fuzzy rough sets," IEEE Transactions on Fuzzy Systems, vol. 21, no. 3, pp. 527540, 2013.

[57] Y. Han, P. Shi, and S. Chen, "Bipolar-Valued Rough Fuzzy Set and Its Applications to the Decision Information System," IEEE Transactions on Fuzzy Systems, vol. 23, no. 6, pp. 2358-2370, 2015.

[58] M. Aggarwal, "Probabilistic variable precision fuzzy rough sets," IEEE Transactions on Fuzzy Systems, vol. 24, no. 1, pp. 29-39, 2016.

[59] D. C. Liang, D. Liu, W. Pedrycz, and P. Hu, "Triangular fuzzy decision-theoretic rough sets," International Journal of Approximate Reasoning, vol. 54, no. 8, pp. 1087-1106, 2013.

[60] Q. Hu, D. Yu, and M. Guo, "Fuzzy preference based rough sets," Information Sciences. An International Journal, vol. 180, no. 10, pp. 2003-2022, 2010.

[61] D. Liang and D. Liu, "Deriving three-way decisions from intuitionistic fuzzy decision-theoretic rough sets," Information Sciences. An International Journal, vol. 300, pp. 28-48, 2015.

[62] B. Sun, W. Ma, and H. Zhao, "A fuzzy rough set approach to emergency material demand prediction over two universes," Applied Mathematical Modelling. Simulation and Computation for Engineering and Environmental Systems, vol. 37, no. 10-11, pp. 7062-7070, 2013.

[63] H. Zhang, L. Shu, and S. Liao, "Hesitant fuzzy rough set over two universes and its application in decision making," Soft Computing, vol. 21, no. 7, pp. 1803-1816, 2017.

[64] J. Zhan and K. Zhu, "A novel soft rough fuzzy set: Z-soft rough fuzzy ideals of hemirings and corresponding decision making," Soft Computing, vol. 21, no. 8, pp. 1923-1936, 2017.

[65] B. Sun and W. Ma, "Soft fuzzy rough sets and its application in decision making," Artificial Intelligence Review, vol. 41, no. 1, pp. 67-68, 2014.

[66] H. L. Yang, S. G. Li, Z. L. Guo, and C. H. Ma, "Transformation of bipolar fuzzy rough set models," Knowledge-Based Systems, vol. 27, pp. 60-68, 2012.

[67] B. Sun, W. Ma, and X. Chen, "Fuzzy rough set on probabilistic approximation space over two universes and its application to emergency decision-making," Expert Systems, vol. 32, no. 4, pp. 507-521, 2015.

[68] T.-F. Fan, C.-J. Liau, and D.-R. Liu, "Dominance-based fuzzy rough set analysis of uncertain and possibilistic data tables," International Journal of Approximate Reasoning, vol. 52, no. 9, pp. 1283-1297, 2011.

[69] Y. Cheng, "The incremental method for fast computing the rough fuzzy approximations," Data and Knowledge Engineering, vol. 70, no. 1, pp. 84-100, 2011.

[70] H.-L. Yang, X. Liao, S. Wang, and J. Wang, "Fuzzy probabilistic rough set model on two universes and its applications," International Journal of Approximate Reasoning, vol. 54, no. 9, pp. 1410-1420, 2013.

[71] W.-Z. Wu, Y. Leung, and M.-W. Shao, "Generalized fuzzy rough approximation operators determined by fuzzy implicators," International Journal of Approximate Reasoning, vol. 54, no. 9, pp. 1388-1409, 2013.
[72] B. Fan, E. C. C. Tsang, W. Xu, and J. Yu, "Double-quantitative rough fuzzy set based decisions: A logical operations method," Information Sciences, vol. 378, pp. 264-281, 2017.

[73] B. Sun, W. Ma, and D. Chen, "Rough approximation of a fuzzy concept on a hybrid attribute information system and its uncertainty measure," Information Sciences. An International Journal, vol. 284, pp. 60-80, 2014.

[74] A. A. Estaji, S. Khodaii, and S. Bahrami, "On rough set and fuzzy sublattice," Information Sciences. An International Journal, vol. 181, no. 18, pp. 3981-3994, 2011.

[75] F. Li and Y. Yin, "The 9 -lower and T-upper fuzzy rough approximation operators on a semigroup," Information Sciences. An International Journal, vol. 195, pp. 241-255, 2012.

[76] Q. Zhang, J. Wang, G. Wang, and H. Yu, "The approximation set of a vague set in rough approximation space," Information Sciences. An International Journal, vol. 300, pp. 1-19, 2015.

[77] Z. Li and R. Cui, "Similarity of fuzzy relations based on fuzzy topologies induced by fuzzy rough approximation operators," Information Sciences, vol. 305, pp. 219-233, 2015.

[78] W.-Z. Wu, Y.-H. Xu, M.-W. Shao, and G. Wang, "Axiomatic characterizations of (S, T)-fuzzy rough approximation operators," Information Sciences, vol. 334-335, pp. 17-43, 2016.

[79] J. Hao and Q. Li, "The relationship between L-fuzzy rough set and L-topology," Fuzzy Sets and Systems. An International Journal in Information Science and Engineering, vol. 178, pp. 7483, 2011.

[80] Y. Cheng, "Forward approximation and backward approximation in fuzzy rough sets," Neurocomputing, vol. 148, pp. 340-353, 2015.

[81] F. Feng, C. Li, B. Davvaz, and M. I. Ali, "Soft sets combined with fuzzy sets and rough sets: a tentative approach," Soft Computing, vol. 14, no. 9, pp. 899-911, 2010.

[82] B. Sun, W. Ma, and H. Zhao, "Decision-theoretic rough fuzzy set model and application," Information Sciences. An International Journal, vol. 283, pp. 180-196, 2014.

[83] R. Jensen, A. Tuson, and Q. Shen, "Finding rough and fuzzyrough set reducts with SAT, Information Sciences. An International Journal, vol. 255, pp. 100-120, 2014.

[84] Y. Qian, Q. Wang, H. Cheng, J. Liang, and C. Dang, "Fuzzyrough feature selection accelerator," Fuzzy Sets and Systems. An International Journal in Information Science and Engineering, vol. 258, pp. 61-78, 2015.

[85] Q. Hu, S. An, and D. Yu, "Soft fuzzy rough sets for robust feature evaluation and selection," Information Sciences. An International Journal, vol. 180, no. 22, pp. 4384-4400, 2010.

[86] T.-P. Hong, L.-H. Tseng, and B.-C. Chien, "Mining from incomplete quantitative data by fuzzy rough sets," Expert Systems with Applications, vol. 37, no. 3, pp. 2644-2653, 2010.

[87] A. Onan, "A fuzzy-rough nearest neighbor classifier combined with consistency-based subset evaluation and instance selection for automated diagnosis of breast cancer," Expert Systems with Applications, vol. 42, no. 20, pp. 6844-6852, 2015.

[88] J. Derrac, C. Cornelis, S. García, and F. Herrera, "Enhancing evolutionary instance selection algorithms by means of fuzzy rough set based feature selection," Information Sciences, vol. 186, no. 1, pp. 73-92, 2012.

[89] R. Jensen and N. Mac Parthaláin, "Towards scalable fuzzyrough feature selection," Information Sciences, vol. 323, Article ID 11619, pp. 1-15, 2015.

[90] S. K. Pal, S. K. Meher, and S. Dutta, "Class-dependent roughfuzzy granular space, dispersion index and classification," Pattern Recognition, vol. 45, no. 7, pp. 2690-2707, 2012. 
[91] P. Maji and P. Garai, "On fuzzy-rough attribute selection: Criteria of Max-Dependency, Max-Relevance, Min-Redundancy, and Max-Significance," Applied Soft Computing Journal, vol. 13, no. 9, pp. 3968-3980, 2013.

[92] A. Ganivada, S. S. Ray, and S. K. Pal, "Fuzzy rough sets, and a granular neural network for unsupervised feature selection," Neural Networks, vol. 48, pp. 91-108, 2013.

[93] P. K. Kumar, P. Vadakkepat, and L. A. Poh, "Fuzzy-rough discriminative feature selection and classification algorithm, with application to microarray and image datasets," Applied Soft Computing Journal, vol. 11, no. 4, pp. 3429-3440, 2011.

[94] Q. Hu, D. Yu, W. Pedrycz, and D. Chen, "Kernelized fuzzy rough sets and their applications," IEEE Transactions on Knowledge and Data Engineering, vol. 23, no. 11, pp. 1649-1667, 2011.

[95] C. Cornelis, J. Medina, and N. Verbiest, "Multi-adjoint fuzzy rough sets: definition, properties and attribute selection," International Journal of Approximate Reasoning, vol. 55, no. 1, part 4, pp. 412-426, 2014.

[96] N. Mac Parthaláin and R. Jensen, "Unsupervised fuzzy-rough set-based dimensionality reduction," Information Sciences, vol. 229, pp. 106-121, 2013.

[97] J. Dai and Q. Xu, "Attribute selection based on information gain ratio in fuzzy rough set theory with application to tumor classification," Applied Soft Computing Journal, vol. 13, no. 1, pp. 211-221, 2013.

[98] D. Chen, Q. He, and X. Wang, "FRSVMs: fuzzy rough set based support vector machines," Fuzzy Sets and Systems. An International Journal in Information Science and Engineering, vol. 161, no. 4, pp. 596-607, 2010.

[99] Q. Hu, S. An, X. Yu, and D. Yu, "Robust fuzzy rough classifiers," Fuzzy Sets and Systems. An International Journal in Information Science and Engineering, vol. 183, pp. 26-43, 2011.

[100] W. Wei, J. Cui, J. Liang, and J. Wang, "Fuzzy rough approximations for set-valued data," Information Sciences, vol. 360, pp. 181201, 2016.

[101] Y. Yao, J. Mi, and Z. Li, "A novel variable precision $(\theta, \sigma)$ fuzzy rough set model based on fuzzy granules," Fuzzy Sets and Systems, vol. 236, pp. 58-72, 2014.

[102] D. Chen, L. Zhang, S. Zhao, Q. Hu, and P. Zhu, "A novel algorithm for finding reducts with fuzzy rough sets," IEEE Transactions on Fuzzy Systems, vol. 20, no. 2, pp. 385-389, 2012.

[103] S. Zhao, H. Chen, C. Li, M. Zhai, and X. Du, "RFRR: Robust fuzzy rough reduction," IEEE Transactions on Fuzzy Systems, vol. 21, no. 5, pp. 825-841, 2013.

[104] D. Chen and Y. Yang, "Attribute reduction for heterogeneous data based on the combination of classical and fuzzy rough set models," IEEE Transactions on Fuzzy Systems, vol. 22, no. 5, pp. 1325-1334, 2014.

[105] Z. Gong and X. Zhang, "Variable precision intuitionistic fuzzy rough sets model and its application," International Journal of Machine Learning and Cybernetics, vol. 5, no. 2, pp. 263-280, 2014.

[106] B. Huang, Y.-1. Zhuang, H.-x. Li, and D.-k. Wei, "A dominance intuitionistic fuzzy-rough set approach and its applications," Applied Mathematical Modelling. Simulation and Computation for Engineering and Environmental Systems, vol. 37, no. 12-13, pp. 7128-7141, 2013.

[107] Y. Yang and C. Hinde, "A new extension of fuzzy sets using rough sets: R-fuzzy sets," Information Sciences. An International Journal, vol. 180, no. 3, pp. 354-365, 2010.
[108] Y. Cheng, D. Miao, and Q. Feng, "Positive approximation and converse approximation in interval-valued fuzzy rough sets," Information Sciences. An International Journal, vol. 181, no. 11, pp. 2086-2110, 2011.

[109] Z. Zhang, "Generalized intuitionistic fuzzy rough sets based on intuitionistic fuzzy coverings," Information Sciences. An International Journal, vol. 198, pp. 186-206, 2012.

[110] Z. Xue, Y. Shang, and A. Feng, "Semi-supervised outlier detection based on fuzzy rough C-means clustering," Mathematics and Computers in Simulation, vol. 80, no. 9, pp. 1911-1921, 2010.

[111] X. Zhang, B. Zhou, and P. Li, "A general frame for intuitionistic fuzzy rough sets," Information Sciences. An International Journal, vol. 216, pp. 34-49, 2012.

[112] W. Wei, J. Liang, Y. Qian, and C. Dang, "Can fuzzy entropies be effective measures for evaluating the roughness of a rough set?" Information Sciences, vol. 232, pp. 143-166, 2013.

[113] B. Huang, C.-x. Guo, Y.-1. Zhuang, H.-x. Li, and X.-z. Zhou, "Intuitionistic fuzzy multigranulation rough sets," Information Sciences. An International Journal, vol. 277, pp. 299-320, 2014.

[114] C. Y. Wang, "Type-2 fuzzy rough sets based on extended tnorms," Information Sciences. An International Journal, vol. 305, pp. 165-183, 2015.

[115] B. Yang and B. Q. Hu, "A fuzzy covering-based rough set model and its generalization over fuzzy lattice," Information Sciences, vol. 367-368, pp. 463-486, 2016.

[116] J. Lu, D. Y. Li, Y. H. Zhai, H. Li, and H. X. Bai, "A model for type2 fuzzy rough sets," Information Sciences, vol. 328, pp. 359-377, 2016.

[117] D. Chen, S. Kwong, Q. He, and H. Wang, "Geometrical interpretation and applications of membership functions with fuzzy rough sets," Fuzzy Sets and Systems. An International Journal in Information Science and Engineering, vol. 193, pp. 122-135, 2012.

[118] L. D'eer, N. Verbiest, C. Cornelis, and L. s. Godo, "A comprehensive study of implicator-conjunctor-based and noisetolerant fuzzy rough sets: definitions, properties and robustness analysis," Fuzzy Sets and Systems. An International Journal in Information Science and Engineering, vol. 275, pp. 1-38, 2015.

[119] L. Ma, "Two fuzzy covering rough set models and their generalizations over fuzzy lattices," Fuzzy Sets and Systems. An International Journal in Information Science and Engineering, vol. 294, pp. 1-17, 2016.

[120] Q. He, C. Wu, D. Chen, and S. Zhao, "Fuzzy rough set based attribute reduction for information systems with fuzzy decisions," Knowledge-Based Systems, vol. 24, no. 5, pp. 689-696, 2011.

[121] H.-L. Yang, S.-G. Li, S. Wang, and J. Wang, "Bipolar fuzzy rough set model on two different universes and its application," Knowledge-Based Systems, vol. 35, pp. 94-101, 2012.

[122] Z. Zhang, "On interval type-2 rough fuzzy sets," KnowledgeBased Systems, vol. 35, pp. 1-13, 2012.

[123] H. Bai, Y. Ge, J. Wang, D. Li, Y. Liao, and X. Zheng, "A method for extracting rules from spatial data based on rough fuzzy sets," Knowledge-Based Systems, vol. 57, pp. 28-40, 2014.

[124] X. R. Zhao and B. Q. Hu, "Fuzzy probabilistic rough sets and their corresponding three-way decisions," Knowledge-Based Systems, vol. 91, pp. 126-142, 2016.

[125] B. Huang, C.-X. Guo, H.-X. Li, G.-F. Feng, and X.-Z. Zhou, "An intuitionistic fuzzy graded covering rough set," KnowledgeBased Systems, vol. 107, pp. 155-178, 2016.

[126] A. S. Khuman, Y. Yang, and R. John, "Quantification of R-fuzzy sets," Expert Systems with Applications, vol. 55, pp. 374-387, 2016. 
[127] Y. Liu and Y. Lin, "Intuitionistic fuzzy rough set model based on conflict distance and applications," Applied Soft Computing Journal, vol. 31, pp. 266-273, 2015.

[128] B. Sun, W. Ma, and Q. Liu, "An approach to decision making based on intuitionistic fuzzy rough sets over two universes," Journal of the Operational Research Society, vol. 64, no. 7, pp. 1079-1089, 2013.

[129] H. Zhang, L. Shu, S. Liao, and C. Xiawu, "Dual hesitant fuzzy rough set and its application," Soft Computing, vol. 21, no. 12, pp. 3287-3305, 2015.

[130] B. Q. Hu, "Generalized interval-valued fuzzy variable precision rough sets determined by fuzzy logical operators," International Journal of General Systems, vol. 44, no. 7-8, pp. 849-875, 2015.

[131] T. Zhao and J. Xiao, "General type-2 fuzzy rough sets based on $\alpha$-plane representation theory," Soft Computing, vol. 18, no. 2, pp. 227-237, 2014.

[132] X. B. Yang, X. N. Song, Y. S. Qi, and J. Y. Yang, "Constructive and axiomatic approaches to hesitant fuzzy rough set," Soft Computing, vol. 18, no. 6, pp. 1067-1077, 2014.

[133] H. D. Zhang, L. Shu, and S. L. Liao, "On interval-valued hesitant fuzzy rough approximation operators," Soft Computing, 2014.

[134] C. Y. Wang and B. Q. Hu, "On fuzzy-valued operations and fuzzy-valued fuzzy sets," Fuzzy Sets and Systems. An International Journal in Information Science and Engineering, vol. 268, pp. 72-92, 2015.

[135] S. Mitra, W. Pedrycz, and B. Barman, "Shadowed c-means: integrating fuzzy and rough clustering," Pattern Recognition, vol. 43, no. 4, pp. 1282-1291, 2010.

[136] C. Chen, N. M. Parthaláin, Y. Li, C. Price, C. Quek, and Q. Shen, "Rough-fuzzy rule interpolation," Information Sciences, vol. 351, pp. 1-17, 2016.

[137] C. Zhang, D. Li, Y. Mu, and D. Song, "An interval-valued hesitant fuzzy multigranulation rough set over two universes model for steam turbine fault diagnosis," Applied Mathematical Modelling. Simulation and Computation for Engineering and Environmental Systems, vol. 42, pp. 693-704, 2017.

[138] F. Bobillo and U. Straccia, "Generalized fuzzy rough description logics," Information Sciences. An International Journal, vol. 189, pp. 43-62, 2012.

[139] G. Liu, "Using one axiom to characterize rough set and fuzzy rough set approximations," Information Sciences. An International Journal, vol. 223, pp. 285-296, 2013.

[140] S. An, H. Shi, Q. Hu, X. Li, and J. Dang, "Fuzzy rough regression with application to wind speed prediction," Information Sciences. An International Journal, vol. 282, pp. 388-400, 2014.

[141] L. S. Riza, A. Janusz, C. Bergmeir et al., "Implementing algorithms of rough set theory and fuzzy rough set theory in the $\mathrm{R}$ package "roughSets", Information Sciences, vol. 287, pp. 68-89, 2014.

[142] R. K. Shiraz, V. Charles, and L. Jalalzadeh, "Fuzzy rough DEA model: A possibility and expected value approaches," Expert Systems with Applications, vol. 41, no. 2, pp. 434-444, 2014.

[143] A. Zeng, T. Li, J. Hu, H. Chen, and C. Luo, "Dynamical updating fuzzy rough approximations for hybrid data under the variation of attribute values," Information Sciences. An International Journal, vol. 378, pp. 363-388, 2017.

[144] J. Zhou, W. Pedrycz, and D. Miao, "Shadowed sets in the characterization of rough-fuzzy clustering," Pattern Recognition, vol. 44, no. 8, pp. 1738-1749, 2011.

[145] N. Verbiest, C. Cornelis, and F. Herrera, "FRPS: A Fuzzy Rough Prototype Selection method," Pattern Recognition, vol. 46, no. 10, pp. 2770-2782, 2013.
[146] S. Pramanik, D. K. Jana, and M. Maiti, "Bi-criteria solid transportation problem with substitutable and damageable items in disaster response operations on fuzzy rough environment," Socio-Economic Planning Sciences, vol. 55, pp. 1-13, 2016.

[147] G. Liu, "Rough set theory based on two universal sets and its applications," Knowledge-Based Systems, vol. 23, no. 2, pp. 110$115,2010$.

[148] S. K. Meher, "Explicit rough-fuzzy pattern classification model," Pattern Recognition Letters, vol. 36, no. 1, pp. 54-61, 2014.

[149] S. Kundu and S. K. Pal, "Fuzzy-rough community in social networks," Pattern Recognition Letters, vol. 67, pp. 145-152, 2015.

[150] A. Ganivada, S. S. Ray, and S. . Pal, "Fuzzy rough granular selforganizing map and fuzzy rough entropy," Theoretical Computer Science, vol. 466, pp. 37-63, 2012.

[151] M. Amiri and R. Jensen, "Missing data imputation using fuzzyrough methods," Neurocomputing, vol. 205, pp. 152-164, 2016.

[152] E. Ramentol, I. Gondres, S. Lajes et al., "Fuzzy-rough imbalanced learning for the diagnosis of High Voltage Circuit Breaker maintenance: The SMOTE-FRST-2T algorithm," Engineering Applications of Artificial Intelligence, vol. 48, pp. 134-139, 2016.

[153] C. Affonso, R. J. Sassi, and R. M. Barreiros, "Biological image classification using rough-fuzzy artificial neural network," Expert Systems with Applications, vol. 42, no. 24, pp. 9482-9488, 2015.

[154] N. Shukla and S. Kiridena, "A fuzzy rough sets-based multiagent analytics framework for dynamic supply chain configuration," International Journal of Production Research, vol. 54, pp. 6984-6996, 2016.

[155] P. Pahlavani, H. Amini Amirkolaee, and S. Talebi Nahr, "A new feature selection from lidar data and digital aerial images acquired for an urban/c environment using an ANFIS-based classification and a fuzzy rough set method," International Journal of Remote Sensing, vol. 36, no. 14, pp. 3587-3615, 2015.

[156] H. Xie and B. Q. Hu, "New extended patterns of fuzzy rough set models on two universes," International Journal of General Systems, vol. 43, no. 6, pp. 570-585, 2014.

[157] S. Zhao, E. C. C. Tsang, D. Chen, and X. Wang, "Building a rulebased classifiera fuzzy-rough set approach," IEEE Transactions on Knowledge and Data Engineering, vol. 22, no. 5, pp. 624-638, 2010.

[158] P. Maji and S. K. Pal, "Fuzzy-rough sets for information measures and selection of relevant genes from microarray data," IEEE Transactions on Systems, Man, and Cybernetics, Part B: Cybernetics, vol. 40, no. 3, pp. 741-752, 2010.

[159] H.-H. Huang and Y.-H. Kuo, "Cross-lingual document representation and semantic similarity measure: A fuzzy set and rough set based approach," IEEE Transactions on Fuzzy Systems, vol. 18, no. 6, pp. 1098-1111, 2010.

[160] R. Wang, D. Chen, and S. Kwong, "Fuzzy-Rough-Set-Based Active Learning," IEEE Transactions on Fuzzy Systems, vol. 22, no. 6, pp. 1699-1704, 2014.

[161] E. Ramentol, S. Vluymans, N. Verbiest et al., "IFROWANN: Imbalanced Fuzzy-Rough Ordered Weighted Average Nearest Neighbor Classification," IEEE Transactions on Fuzzy Systems, vol. 23, no. 5, pp. 1622-1637, 2015.

[162] X. F. Zhang, G. Yu, Z. Y. Hu, C. H. Pei, and G. Q. Ma, "Parallel disassembly sequence planning for complex products based on fuzzy-rough sets," International Journal of Advanced Manufacturing Technology, vol. 72, no. 1-4, pp. 231-239, 2014.

[163] J. Derrac, N. Verbiest, S. García, C. Cornelis, and F. Herrera, “On the use of evolutionary feature selection for improving fuzzy 
rough set based prototype selection," Soft Computing, vol. 17, no. 2, pp. 223-238, 2013.

[164] N. Verbiest, E. Ramentol, C. Cornelis, and F. Herrera, "Preprocessing noisy imbalanced datasets using SMOTE enhanced with fuzzy rough prototype selection," Applied Soft Computing, vol. 22, pp. 511-517, 2014.

[165] J.-H. Zhai, "Fuzzy decision tree based on fuzzy-rough technique," Soft Computing, vol. 15, no. 6, pp. 1087-1096, 2011.

[166] J. Zhao, Z. Zhang, C. Han, and Z. Zhou, "Complement information entropy for uncertainty measure in fuzzy rough set and its applications," Soft Computing, vol. 19, no. 7, pp. 1997-2010, 2015.

[167] Q. Hu, L. Zhang, S. An, D. Zhang, and D. Yu, "On robust fuzzy rough set models," IEEE Transactions on Fuzzy Systems, vol. 20, no. 4, pp. 636-651, 2012.

[168] C. Changdar, R. K. Pal, and G. S. Mahapatra, "A genetic ant colony optimization based algorithm for solid multiple travelling salesmen problem in fuzzy rough environment," Soft Computing, pp. 1-15, 2016.

[169] B. Sun and W. Ma, "An approach to evaluation of emergency plans for unconventional emergency events based on soft fuzzy rough set," Kybernetes. The International Journal of Cybernetics, Systems and Management Sciences, vol. 45, no. 3, pp. 461-473, 2016.

[170] W. S. Du and B. Q. Hu, "Dominance-based rough fuzzy set approach and its application to rule induction," European Journal of Operational Research, vol. 261, no. 2, pp. 690-703, 2017.

[171] J. Hu, T. Li, C. Luo, H. Fujita, and S. Li, "Incremental fuzzy probabilistic rough sets over two universes," International Journal of Approximate Reasoning, vol. 81, pp. 28-48, 2017.

[172] D. Liang, Z. Xu, and D. Liu, "Three-way decisions with intuitionistic fuzzy decision-theoretic rough sets based on point operators," Information Sciences, vol. 375, pp. 183-201, 2017.

[173] D. Liang, Z. Xu, and D. Liu, "Three-way decisions based on decision-theoretic rough sets with dual hesitant fuzzy information," Information Sciences, vol. 396, pp. 127-143, 2017.

[174] J. Qiao and B. Q. Hu, "Granular variable precision $L$-fuzzy rough sets based on residuated lattices," Fuzzy Sets and Systems, 2016.

[175] J. Qiao and B. Q. Hu, "On $(\odot, \&)$-fuzzy rough sets based on residuated and coresiduated lattices," Fuzzy Sets and Systems, 2017.

[176] J. Shi, Y. Lei, Y. Zhou, and M. Gong, "Enhanced rough-fuzzy cmeans algorithm with strict rough sets properties," Applied Soft Computing Journal, vol. 46, pp. 827-850, 2016.

[177] C. Y. Wang, "Notes on a comprehensive study of implicatorconjunctor-based and noise-tolerant fuzzy rough sets: definitions, properties and robustness analysis," Fuzzy Sets and Systems. An International Journal in Information Science and Engineering, vol. 294, pp. 36-43, 2016.

[178] B. Yang and B. Q. Hu, "On some types of fuzzy covering-based rough sets," Fuzzy Sets and Systems, vol. 312, pp. 36-65, 2017.

[179] N. N. Morsi and M. M. Yakout, "Axiomatics for fuzzy rough sets," Fuzzy Sets and Systems. An International Journal in Information Science and Engineering, vol. 100, no. 1-3, pp. 327342, 1998.

[180] X. Wang and J. Hong, "Learning optimization in simplifying fuzzy rules," Fuzzy Sets and Systems. An International Journal in Information Science and Engineering, vol. 106, no. 3, pp. 349356, 1999.

[181] D. Molodtsov, "Soft set theory-first results," Computers \& Mathematics with Applications, vol. 37, no. 4-5, pp. 19-31, 1999.
[182] P. K. Maji, R. Biswas, and A. R. Roy, "Fuzzy soft sets," Journal of Fuzzy Mathematics, vol. 9, no. 3, pp. 589-602, 2001.

[183] M. De Cock, C. Cornelis, and E. Kerre, "Fuzzy rough sets: Beyond the obvious," in Proceedings of the 2004 IEEE International Conference on Fuzzy Systems - Proceedings, pp. 103-108, hun, July 2004.

[184] R. Jensen and Q. Shen, "Fuzzy-rough attribute reduction with application to web categorization," Fuzzy Sets and Systems. An International Journal in Information Science and Engineering, vol. 141, no. 3, pp. 469-485, 2004.

[185] A. Mieszkowicz-Rolka and L. Rolka, "Variable precision fuzzy rough sets," Lecture Notes in Computer Science (including subseries Lecture Notes in Artificial Intelligence and Lecture Notes in Bioinformatics), vol. 3100, pp. 144-160, 2004.

[186] J.-S. Mi and W.-X. Zhang, "An axiomatic characterization of a fuzzy generalization of rough sets," Information Sciences. An International Journal, vol. 160, no. 1-4, pp. 235-249, 2004.

[187] Q. Shen and R. Jensen, "Selecting informative features with fuzzy-rough sets and its application for complex systems monitoring," Pattern Recognition, vol. 37, no. 7, pp. 1351-1363, 2004.

[188] W.-Z. Wu, Y. Leung, and J.-S. Mi, "On characterizations of (I,T)fuzzy rough approximation operators," Fuzzy Sets and Systems, vol. 154, no. 1, pp. 76-102, 2005.

[189] R. B. Bhatt and M. Gopal, "On the compact computational domain of fuzzy-rough sets," Pattern Recognition Letters, vol. 26, no. 11, pp. 1632-1640, 2005.

[190] D. S. Yeung, D. Chen, E. C. C. Tsang, J. W. T. Lee, and W. Xizhao, "On the generalization of fuzzy rough sets," IEEE Transactions on Fuzzy Systems, vol. 13, no. 3, pp. 343-361, 2005.

[191] T. Deng, Y. Chen, W. Xu, and Q. Dai, "A novel approach to fuzzy rough sets based on a fuzzy covering," Information Sciences. An International Journal, vol. 177, no. 11, pp. 2308-2326, 2007.

[192] T. Li and J. Ma, "Fuzzy Approximation Operators Based on Coverings," in Rough Sets, Fuzzy Sets, Data Mining and Granular Computing, vol. 4482 of Lecture Notes in Computer Science, pp. 55-62, Springer Berlin Heidelberg, Berlin, Heidelberg, 2007.

[193] H. Aktaş and N. Çağman, "Soft sets and soft groups," Information Sciences, vol. 177, no. 13, pp. 2726-2735, 2007.

[194] S. Greco, R. Slowinski, and Y. Yao, "Bayesian decision theory for dominance-based rough set approach," in Proceeding of the International Conference on Rough Sets and Knowledge Technology, pp. 134-141, Springer, 2007.

[195] C. Cornelis, R. Jensen, G. Hurtado, and D. Ślęzak, "Attribute selection with fuzzy decision reducts," Information Sciences, vol. 180, no. 2, pp. 209-224, 2010.

[196] X. Wang, E. C. Tsang, S. Zhao, D. Chen, and D. S. Yeung, "Learning fuzzy rules from fuzzy samples based on rough set technique," Information Sciences, vol. 177, no. 20, pp. 4493-4514, 2007.

[197] Q. Hu, Z. Xie, and D. Yu, "Hybrid attribute reduction based on a novel fuzzy-rough model and information granulation," Pattern Recognition, vol. 40, no. 12, pp. 3509-3521, 2007.

[198] P. Lingras, M. Chen, and D. Miao, "Rough multi-category decision theoretic framework in," in International Conference on Rough Sets and Knowledge Technology, pp. 676-683, Springer, 2008.

[199] P. Lingras, M. Chen, and D. Miao, "Rough cluster quality index based on decision theory," IEEE Transactions on Knowledge and Data Engineering, vol. 21, no. 7, pp. 1014-1026, 2009.

[200] Y.-H. She and G.-J. Wang, "An axiomatic approach of fuzzy rough sets based on residuated lattices," Computers and Mathematics with Applications, vol. 58, no. 1, pp. 189-201, 2009. 
[201] S. Zhao, E. C. C. Tsang, and D. Chen, "The model of fuzzy variable precision rough sets," IEEE Transactions on Fuzzy Systems, vol. 17, no. 2, pp. 451-467, 2009.

[202] H. Y. Wu, Y. Y. Wu, and J. P. Luo, "An interval type-2 fuzzy rough set model for attribute reduction," IEEE Transactions on Fuzzy Systems, vol. 17, no. 2, pp. 301-315, 2009.

[203] R. Yan, J. Zheng, J. Liu, and Y. Zhai, "Research on the model of rough set over dual-universes," Knowledge-Based Systems, vol. 23, no. 8, pp. 817-822, 2010.

[204] X. Yang and J. Yao, "A multi-agent decision-theoretic rough set model," Lecture Notes in Computer Science (including subseries Lecture Notes in Artificial Intelligence and Lecture Notes in Bioinformatics), vol. 6401, pp. 711-718, 2010.

[205] W. Xu, S. Liu, Q. Wang, and W. Zhang, "The first type of graded rough set based on rough membership function," in Proceedings of the 2010 7th International Conference on Fuzzy Systems and Knowledge Discovery, FSKD 2010, pp. 1922-1926, chn, August 2010.

[206] M. Xia and Z. Xu, "Generalized point operators for aggregating intuitionistic fuzzy information," International Journal of Intelligent Systems, vol. 25, pp. 1061-1080, 2010.

[207] W.-Z. Wu, "On some mathematical structures of T-fuzzy rough set algebras in infinite universes of discourse," Fundamenta Informaticae, vol. 108, no. 3-4, pp. 337-369, 2011.

[208] F. Feng, X. Liu, V. Leoreanu-Fotea, and Y. B. Jun, "Soft sets and soft rough sets," Information Sciences, vol. 181, no. 6, pp. 11251137, 2011.

[209] D. Meng, X. Zhang, and K. Qin, "Soft rough fuzzy sets and soft fuzzy rough sets," Computers \& Mathematics with Applications, vol. 62, no. 12, pp. 4635-4645, 2011.

[210] H. Li and X. Zhou, "Risk decision making based on decisiontheoretic rough set: A three-way view decision model," International Journal of Computational Intelligence Systems, vol. 4, no. 1, pp. 1-11, 2011.

[211] H. Li, X. Zhou, J. Zhao, and D. Liu, "Attribute reduction in decision-theoretic rough set model: A further investigation," Lecture Notes in Computer Science (including subseries Lecture Notes in Artificial Intelligence and Lecture Notes in Bioinformatics), vol. 6954, pp. 466-475, 2011.

[212] X. Jia, W. Li, L. Shang, and J. Chen, "An optimization viewpoint of decision-theoretic rough set model," Lecture Notes in Computer Science (including subseries Lecture Notes in Artificial Intelligence and Lecture Notes in Bioinformatics), vol. 6954, pp. 457-465, 2011.

[213] D. Liu, T. Li, and D. Ruan, "Probabilistic model criteria with decision-theoretic rough sets," Information Sciences. An International Journal, vol. 181, no. 17, pp. 3709-3722, 2011.

[214] D. Liu, Y. Yao, and T. Li, "Three-way investment decisions with decision-theoretic rough sets," International Journal of Computational Intelligence Systems, vol. 4, no. 1, pp. 66-74, 2011.

[215] C. Degang, Y. Yongping, and W. Hui, "Granular computing based on fuzzy similarity relations," Soft Computing, vol. 15, no. 6, pp. 1161-1172, 2011.

[216] W. Ma and B. Sun, "On relationship between probabilistic rough set and Bayesian risk decision over two universes," International Journal of General Systems, vol. 41, no. 3, pp. 225-245, 2012.

[217] W. Ma and B. Sun, "Probabilistic rough set over two universes and rough entropy," International Journal of Approximate Reasoning, vol. 53, no. 4, pp. 608-619, 2012.

[218] C. Liu, D. Miao, and N. Zhang, "Graded rough set model based on two universes and its properties," Knowledge-Based Systems, vol. 33, pp. 65-72, 2012.
[219] W. Wei, J. Liang, and Y. Qian, "A comparative study of rough sets for hybrid data," Information Sciences. An International Journal, vol. 190, pp. 1-16, 2012.

[220] Z. M. Ma and B. Q. Hu, "Topological and lattice structures of L-fuzzy rough sets determined by lower and upper sets," Information Sciences. An International Journal, vol. 218, pp. 194204, 2013.

[221] X. Zhang and D. Miao, “Two basic double-quantitative rough set models of precision and grade and their investigation using granular computing," International Journal of Approximate Reasoning, vol. 54, no. 8, pp. 1130-1148, 2013.

[222] X. Zhang and D. Miao, "Quantitative information architecture, granular computing and rough set models in the doublequantitative approximation space of precision and grade," Information Sciences. An International Journal, vol. 268, pp. 147-168, 2014.

[223] D. Liu, T. Li, and D. Liang, "Incorporating logistic regression to decision-theoretic rough sets for classifications," International Journal of Approximate Reasoning, vol. 55, no. 1, part 2, pp. 197210, 2014.

[224] X. Ma, G. Wang, H. Yu, and T. Li, "Decision region distribution preservation reduction in decision-theoretic rough set model," Information Sciences. An International Journal, vol. 278, pp. 614640, 2014.

[225] Y. Qian, H. Zhang, Y. Sang, and J. Liang, "Multigranulation decision-theoretic rough sets," International Journal of Approximate Reasoning, vol. 55, no. 1, part 2, pp. 225-237, 2014.

[226] X. Zhang and D. Miao, "Reduction target structure-based hierarchical attribute reduction for two-category decision-theoretic rough sets," Information Sciences. An International Journal, vol. 277, pp. 755-776, 2014.

[227] X. Zhang and D. Miao, "Region-based quantitative and hierarchical attribute reduction in the two-category decision theoretic rough set model," Knowledge-Based Systems, vol. 71, pp. 146-161, 2014.

[228] X. Zhang and D. Miao, "An expanded double-quantitative model regarding probabilities and grades and its hierarchical double-quantitative attribute reduction," Information Sciences. An International Journal, vol. 299, pp. 312-336, 2015.

[229] Z. Li and R. Cui, "T-similarity of fuzzy relations and related algebraic structures," Fuzzy Sets and Systems. An International Journal in Information Science and Engineering, vol. 275, pp. 130-143, 2015.

[230] W. Xu, W. Li, and S. Luo, "Knowledge reductions in generalized approximation space over two universes based on evidence theory," Journal of Intelligent and Fuzzy Systems, vol. 28, no. 6, pp. 2471-2480, 2015.

[231] W. Li and W. Xu, "Multigranulation decision-theoretic rough set in ordered information system," Fundamenta Informaticae, vol. 139, no. 1, pp. 67-89, 2015.

[232] D. Liang, W. Pedrycz, D. Liu, and P. Hu, "Three-way decisions based on decision-theoretic rough sets under linguistic assessment with the aid of group decision making," Applied Soft Computing Journal, vol. 29, pp. 256-269, 2015.

[233] H. Ju, X. Yang, P. Yang, H. Li, and X. Zhou, "A moderate attribute reduction approach in decision-theoretic rough set," in Rough sets, fuzzy sets, data mining, and granular computing, vol. 9437 of Lecture Notes in Comput. Sci., pp. 376-388, Springer, Cham, 2015.

[234] W. Li and W. Xu, "Double-quantitative decision-theoretic rough set," Information Sciences, vol. 316, pp. 54-67, 2015. 
[235] H.-R. Zhang and F. Min, "Three-way recommender systems based on random forests," Knowledge-Based Systems, vol. 91, pp. 275-286, 2016.

[236] Y. Zhang and J. Yao, "Gini objective functions for three-way classifications," International Journal of Approximate Reasoning, vol. 81, pp. 103-114, 2017.

[237] B. Sun, W. Ma, and X. Xiao, “Three-way group decision making based on multigranulation fuzzy decision-theoretic rough set over two universes," International Journal of Approximate Reasoning, vol. 81, pp. 87-102, 2017.

[238] J. Qiao and B. Q. Hu, "Granular variable precision L-fuzzy rough sets based on residuated lattices," Fuzzy Sets and Systems, 2015.

[239] D. Moher, A. Liberati, and J. Tetzlaff, "Preferred reporting items for systematic reviews and meta-analyses: the PRISMA statement," Annals of Internal Medicine, vol. 151, pp. 264-269, 2009.

[240] D. Budgen and P. Brereton, "Performing systematic literature reviews in software engineering," in Proceedings of the 28th international conference on Software engineering, pp. 1051-1052, May 2006.

[241] P. J. Phillips and E. M. Newton, "Meta-analysis of face recognition algorithms," in Proceedings of the 5th IEEE International Conference on Automatic Face Gesture Recognition, FGR 2002, pp. 235-241, usa, May 2002.

[242] A. Liberati, D. G. Altman, J. Tetzlaff et al., "The PRISMA statement for reporting systematic reviews and meta-analyses of studies that evaluate health care interventions: explanation and elaboration," Annals of Internal Medicine, vol. 151, no. 4, pp. 65-94, 2009.

[243] A. Hughes-Morley, B. Young, W. Waheed, N. Small, and P. Bower, "Factors affecting recruitment into depression trials: Systematic review, meta-synthesis and conceptual framework," Journal of Affective Disorders, vol. 172, pp. 274-290, 2015.

[244] N. S. Consedine, N. L. Tuck, C. R. Ragin, and B. A. Spencer, "Beyond the black box: a systematic review of breast, prostate, colorectal, and cervical screening among native and immigrant african-descent caribbean populations," Journal of Immigrant and Minority Health, vol. 17, no. 3, pp. 905-924, 2015.

[245] F. Xu, L. Wei, Z. Bi, and L. Zhu, "Research on fuzzy rough parallel reduction based on mutual information," Journal of Computational Information Systems, vol. 10, no. 12, pp. 53915401, 2014.

[246] Y. Yang, Z. Chen, Z. Liang, and G. Wang, "Attribute reduction for massive data based on rough set theory and mapreduce," Lecture Notes in Computer Science (including subseries Lecture Notes in Artificial Intelligence and Lecture Notes in Bioinformatics), vol. 6401, pp. 672-678, 2010.

[247] J. Zhang, T. Li, D. Ruan, Z. Gao, and C. Zhao, "A parallel method for computing rough set approximations," Information Sciences, vol. 194, pp. 209-223, 2012.

[248] J. Zhang, J.-S. Wong, T. Li, and Y. Pan, “A comparison of parallel large-scale knowledge acquisition using rough set theory on different MapReduce runtime systems," International Journal of Approximate Reasoning, vol. 55, no. 3, pp. 896-907, 2014.

[249] Y. Yang and Z. Chen, "Parallelized computing of attribute core based on rough set theory and MapReduce," Lecture Notes in Computer Science (including subseries Lecture Notes in Artificial Intelligence and Lecture Notes in Bioinformatics), vol. 7414, pp. 155-160, 2012.

[250] D. Dubois and H. Prade, "Twofold fuzzy sets and rough sets-some issues in knowledge representation," Fuzzy Sets and
Systems. An International Journal in Information Science and Engineering, vol. 23, no. 1, pp. 3-18, 1987.

[251] "Rough sets, fuzzy sets, data mining and granular computing," in Lecture Notes in Computer Science (including subseries Lecture Notes in Artificial Intelligence and Lecture Notes in Bioinformatics), 13th International Conference (RSFDGrC '11), Moscow, Russia, June 25-27, 2011, S. O. Kuznetsov, D. Ślęzak, D. H. Hepting, and B. G. Mirkin, Eds., vol. 6743, pp. 1-370, 2011.

[252] B. Tripathy, D. Acharjya, and V. Cynthya, "A framework for intelligent medical diagnosis using rough set with formal concept analysis," International Journal of Artificial Intelligence \& Applications, vol. 2, pp. 45-66, 2011.

[253] S. Greco, B. Matarazzo, and R. Slowinski, "Fuzzy dominancebased rough set approach," in Advances in Fuzzy Systems and Intelligent Technologies, F. Masulli, R. Parenti, and G. Pasi, Eds., pp. 56-66, Shaker Publishing, Maastricht, 2000.

[254] S. Greco, M. Inuiguchi, and R. Slowiński, "Possibility and necessity measures in dominance-based rough set approach," in Proceedings of the International Conference on Rough Sets and Current Trends in Computing, pp. 85-92, 2002.

[255] S. Greco, B. Matarazzo, and R. Slowinski, "The use of rough sets and fuzzy sets in MCDM," in Multicriteria Decision Making, pp. 397-455, Springer, 1999.

[256] S. Greco, B. Matarazzo, and R. Slowinski, "Rough approximation of a preference relation by dominance relations," European Journal of Operational Research, vol. 117, no. 1, pp. 63-83, 1999.

[257] S. Chakhar, A. Ishizaka, A. Labib, and I. Saad, "Dominancebased rough set approach for group decisions," European Journal of Operational Research, vol. 251, no. 1, pp. 206-224, 2016.

[258] W. S. Du and B. Q. Hu, "Dominance-based rough set approach to incomplete ordered information systems," Information Sciences. An International Journal, vol. 346/347, pp. 106-129, 2016.

[259] B. Sun and W. Ma, "Rough approximation of a preference relation by multi-decision dominance for a multi-agent conflict analysis problem," Information Sciences. An International Journal, vol. 315, pp. 39-53, 2015.

[260] M. Szeląg, S. Greco, and R. Słowiński, "Variable consistency dominance-based rough set approach to preference learning in multicriteria ranking," Information Sciences, vol. 277, pp. 525552, 2014.

[261] X. Zhang, D. Chen, and E. C. Tsang, "Generalized dominance rough set models for the dominance intuitionistic fuzzy information systems," Information Sciences. An International Journal, vol. 378, pp. 1-25, 2017.

[262] S. Li, T. Li, and D. Liu, "Incremental updating approximations in dominance-based rough sets approach under the variation of the attribute set," Knowledge-Based Systems, vol. 40, pp. 17-26, 2013.

[263] J. Błaszczyński, S. Greco, and R. Słowiński, "Multi-criteria classification - A new scheme for application of dominancebased decision rules," European Journal of Operational Research, vol. 181, no. 3, pp. 1030-1044, 2007.

[264] S. Chakhar and I. Saad, "Dominance-based rough set approach for groups in multicriteria classification problems," Decision Support Systems, vol. 54, no. 1, pp. 372-380, 2012.

[265] M. Inuiguchi, Y. Yoshioka, and Y. Kusunoki, "Variable-precision dominance-based rough set approach and attribute reduction," International Journal of Approximate Reasoning, vol. 50, no. 8, pp. 1199-1214, 2009. 
[266] K. Dembczyński, S. Greco, and R. Słowiński, "Rough set approach to multiple criteria classification with imprecise evaluations and assignments," European Journal of Operational Research, vol. 198, no. 2, pp. 626-636, 2009.

[267] X. Yang, J. Yang, C. Wu, and D. Yu, "Dominance-based rough set approach and knowledge reductions in incomplete ordered information system," Information Sciences. An International Journal, vol. 178, no. 4, pp. 1219-1234, 2008.

[268] S. Li and T. Li, "Incremental update of approximations in dominance-based rough sets approach under the variation of attribute values," Information Sciences. An International Journal, vol. 294, pp. 348-361, 2015.

[269] R. Jensen and Q. Shen, "Fuzzy-rough data reduction with ant colony optimization," Fuzzy Sets and Systems. An International Journal in Information Science and Engineering, vol. 149, no. 1, pp. 5-20, 2005.

[270] C. Wang and F. Ou, "An algorithm for decision tree construction based on rough set theory," in Proceedings of the International Conference on Computer Science and Information Technology (ICCSIT '08), pp. 295-298, September 2008.

[271] H.-L. Chen, B. Yang, J. Liu, and D.-Y. Liu, "A support vector machine classifier with rough set-based feature selection for breast cancer diagnosis," Expert Systems with Applications, vol. 38, no. 7, pp. 9014-9022, 2011.

[272] C. Shang and D. Barnes, "Fuzzy-rough feature selection aided support vector machines for Mars image classification," Computer Vision and Image Understanding, vol. 117, no. 3, pp. 202213, 2013.

[273] Q. He and C. Wu, "Membership evaluation and feature selection for fuzzy support vector machine based on fuzzy rough sets," Soft Computing, vol. 15, no. 6, pp. 1105-1114, 2011.

[274] S. B. Kotsiantis, "Decision trees: A recent overview," Artificial Intelligence Review, vol. 39, no. 4, pp. 261-283, 2013.

[275] L. Rokach and O. Maimon, Data mining with decision trees: theory and applications, World Scientific, Data mining with decision trees, theory and applications, 2014.

[276] X.-Z. Zhu, W. Zhu, and X.-N. Fan, "Rough set methods in feature selection via submodular function," Soft Computing, vol. 21, no. 13, pp. 3699-3711, 2016.

[277] H. H. Inbarani, M. Bagyamathi, and A. T. Azar, "A novel hybrid feature selection method based on rough set and improved harmony search," Neural Computing and Applications, vol. 26, no. 8, pp. 1859-1880, 2015.

[278] G. Beliakov, D. Gómez, S. James, J. Montero, and J. T. Rodríguez, "Approaches to learning strictly-stable weights for data with missing values," Fuzzy Sets and Systems, vol. 325, pp. 97-113, 2017.

[279] S. Jurado, À. Nebot, F. Mugica, and M. Mihaylov, "Fuzzy inductive reasoning forecasting strategies able to cope with missing data: A smart grid application," Applied Soft Computing Journal, vol. 51, pp. 225-238, 2017.

[280] A. Paul, J. Sil, and C. D. Mukhopadhyay, "Gene selection for designing optimal fuzzy rule base classifier by estimating missing value," Applied Soft Computing Journal, vol. 55, pp. 276288, 2017.

[281] L. Zhang, W. Lu, X. Liu, W. Pedrycz, and C. Zhong, "Fuzzy c-means clustering of incomplete data based on probabilistic information granules of missing values," Knowledge-Based Systems, vol. 99, pp. 51-70, 2016.

[282] H. Shidpour, C. Da Cunha, and A. Bernard, "Group multicriteria design concept evaluation using combined rough set theory and fuzzy set theory," Expert Systems with Applications, vol. 64, pp. 633-644, 2016.

[283] C. Bai, D. Dhavale, and J. Sarkis, "Complex investment decisions using rough set and fuzzy c-means: an example of investment in green supply chains," European Journal of Operational Research, vol. 248, no. 2, pp. 507-521, 2016.

[284] M. I. Ali, "A note on soft sets, rough soft sets and fuzzy soft sets," Applied Soft Computing Journal, vol. 11, no. 4, pp. 3329-3332, 2011.

[285] X.-Z. Wang, L.-C. Dong, and J.-H. Yan, "Maximum ambiguitybased sample selection in fuzzy decision tree induction," IEEE Transactions on Knowledge and Data Engineering, vol. 24, no. 8, pp. 1491-1505, 2012.

[286] R. Diao and Q. Shen, "A harmony search based approach to hybrid fuzzy-rough rule induction," in Proceedings of the 2012 IEEE International Conference on Fuzzy Systems, June 2012.

[287] J. Błaszczyński, R. Słowiński, and M. Szeląg, "Sequential covering rule induction algorithm for variable consistency rough set approaches," Information Sciences, vol. 181, no. 5, pp. 987-1002, 2011.

[288] J. Xu and L. Zhao, "A multi-objective decision-making model with fuzzy rough coefficients and its application to the inventory problem," Information Sciences. An International Journal, vol. 180 , no. 5, pp. 679-696, 2010.

[289] R. Jensen and C. Cornelis, "Fuzzy-rough nearest neighbour classification and prediction," Theoretical Computer Science, vol. 412, no. 42, pp. 5871-5884, 2011.

[290] Y. Qu, Q. Shen, N. Mac Parthaláin, C. Shang, and W. Wu, "Fuzzy similarity-based nearest-neighbour classification as alternatives to their fuzzy-rough parallels," International Journal of Approximate Reasoning, vol. 54, no. 1, pp. 184-195, 2013.

[291] M. Sarkar, "Fuzzy-rough nearest neighbor algorithms in classification," Fuzzy Sets and Systems. An International Journal in Information Science and Engineering, vol. 158, no. 19, pp. 21342152, 2007.

[292] R. Jensen and C. Cornelis, "Fuzzy-rough nearest neighbour classification," Lecture Notes in Computer Science (including subseries Lecture Notes in Artificial Intelligence and Lecture Notes in Bioinformatics), vol. 6499, pp. 56-72, 2011.

[293] N. Verbiest, C. Cornelis, and R. Jensen, "Fuzzy rough positive region based nearest neighbour classification," in Proceedings of the 2012 IEEE International Conference on Fuzzy Systems, June 2012.

[294] Z. Zhang, "On characterization of generalized interval type2 fuzzy rough sets," Information Sciences. An International Journal, vol. 219, pp. 124-150, 2013.

[295] Z. Zhang, "An interval-valued intuitionistic fuzzy rough set model," Fundamenta Informaticae, vol. 97, no. 4, pp. 471-498, 2009.

[296] X. Liu, X. Feng, and W. Pedrycz, "Extraction of fuzzy rules from fuzzy decision trees: An axiomatic fuzzy sets (AFS) approach," Data and Knowledge Engineering, vol. 84, pp. 1-25, 2013.

[297] X.-Z. Wang, J.-H. Zhai, and S.-X. Lu, "Induction of multiple fuzzy decision trees based on rough set technique," Information Sciences. An International Journal, vol. 178, no. 16, pp. 3188-3202, 2008.

[298] R. M. Rodríguez, L. Martınez, and F. Herrera, "A group decision making model dealing with comparative linguistic expressions based on hesitant fuzzy linguistic term sets," Information Sciences, vol. 241, pp. 28-42, 2013. 
[299] T. Li, D. Ruan, W. Geert, J. Song, and Y. Xu, "A rough sets based characteristic relation approach for dynamic attribute generalization in data mining," Knowledge-Based Systems, vol. 20, no. 5, pp. 485-494, 2007.

[300] S. P. Tiwari and A. K. Srivastava, "Fuzzy rough sets, fuzzy preorders and fuzzy topologies," Fuzzy Sets and Systems. An International Journal in Information Science and Engineering, vol. 210, pp. 63-68, 2013.

[301] J. Zhang, T. Li, D. Ruan, and D. Liu, "Neighborhood rough sets for dynamic data mining," International Journal of Intelligent Systems, vol. 27, no. 4, pp. 317-342, 2012.

[302] H. Chen, T. Li, C. Luo, S.-J. Horng, and G. Wang, "A DecisionTheoretic Rough Set Approach for Dynamic Data Mining," IEEE Transactions on Fuzzy Systems, vol. 23, no. 6, pp. 19581970, 2015.

[303] M. L. Othman, I. Aris, S. M. Abdullah, M. L. Ali, and M. R. Othman, "Knowledge discovery in distance relay event report: a comparative data-mining strategy of rough set theory with decision tree," IEEE Transactions on Power Delivery, vol. 25, no. 4, pp. 2264-2287, 2010.

[304] Y. H. Hung, "A neural network classifier with rough set-based feature selection to classify multiclass IC package products," Advanced Engineering Informatics, vol. 23, no. 3, pp. 348-357, 2009.

[305] Y. Bi, T. Anderson, and S. McClean, "A rough set model with ontologies for discovering maximal association rules in document collections," Knowledge-Based Systems, vol. 16, no. 56, pp. 243-251, 2003.

[306] A. Formica, "Semantic Web search based on rough sets and fuzzy formal concept analysis," Knowledge-Based Systems, vol. 26, pp. 40-47, 2012.

[307] P. Klinov and L. J. Mazlack, "Fuzzy rough approach to handling imprecision in Semantic Web ontologies," in Proceedings of the NAFIPS 2006 - 2006 Annual Meeting of the North American Fuzzy Information Processing Society, pp. 142-147, June 2006.

[308] Q. Hu, D. Yu, and Z. Xie, "Information-preserving hybrid data reduction based on fuzzy-rough techniques," Pattern Recognition Letters, vol. 27, no. 5, pp. 414-423, 2006.

[309] Q. Shen and A. Chouchoulas, "A rough-fuzzy approach for generating classification rules," Pattern Recognition, vol. 35, no. 11, pp. 2425-2438, 2002.

[310] J.-H. Lee, J. R. Anaraki, C. W. Ahn, and J. An, "Efficient classification system based on Fuzzy-Rough Feature Selection and Multitree Genetic Programming for intension pattern recognition using brain signal," Expert Systems with Applications, vol. 42, no. 3, pp. 1644-1651, 2015.

[311] K. Y. Huang, "An enhanced classification method comprising a genetic algorithm, rough set theory and a modified PBMFindex function," Applied Soft Computing Journal, vol. 12, no. 1, pp. 46-63, 2012.

[312] C.-H. Cheng, T.-L. Chen, and L.-Y. Wei, "A hybrid model based on rough sets theory and genetic algorithms for stock price forecasting," Information Sciences, vol. 180, no. 9, pp. 1610-1629, 2010.

[313] W.-Y. Liang and C.-C. Huang, “The generic genetic algorithm incorporates with rough set theory-an application of the web services composition," Expert Systems with Applications, vol. 36, no. 3, pp. 5549-5556, 2009.

[314] Z. Tao and J. Xu, "A class of rough multiple objective programming and its application to solid transportation problem," Information Sciences. An International Journal, vol. 188, pp. 215235, 2012.
[315] P. Kundu, M. B. Kar, S. Kar, T. Pal, and M. Maiti, "A solid transportation model with product blending and parameters as rough variables," Soft Computing, vol. 21, no. 9, pp. 2297-2306, 2017.

[316] P. Kumar, S. Gupta, and B. Bhasker, "An upper approximation based community detection algorithm for complex networks," Decision Support Systems, vol. 96, pp. 103-118, 2017.

[317] S.-H. Liao and H.-K. Chang, "A rough set-based association rule approach for a recommendation system for online consumers," Information Processing and Management, vol. 52, no. 6, pp. 11421160, 2016.

[318] G. A. Montazer and S. Yarmohammadi, "Detection of phishing attacks in Iranian e-banking using a fuzzy-rough hybrid system," Applied Soft Computing, vol. 35, pp. 482-492, 2015.

[319] R. Li and Z.-O. Wang, "Mining classification rules using rough sets and neural networks," European Journal of Operational Research, vol. 157, no. 2, pp. 439-448, 2004.

[320] X. Pan, S. Zhang, H. Zhang, X. Na, and X. Li, "A variable precision rough set approach to the remote sensing land use/cover classification," Computers \& Geosciences, vol. 36, no. 12, pp. 1466-1473, 2010.

[321] F. Xie, Y. Lin, and W. Ren, "Optimizing model for land use/land cover retrieval from remote sensing imagery based on variable precision rough sets," Ecological Modelling, vol. 222, no. 2, pp. 232-240, 2011.

[322] J. Meng, J. Zhang, R. Li, and Y. Luan, "Gene selection using rough set based on neighborhood for the analysis of plant stress response," Applied Soft Computing Journal, vol. 25, pp. 51-63, 2014.

[323] Y. Chen, Z. Zhang, J. Zheng, Y. Ma, and Y. Xue, "Gene selection for tumor classification using neighborhood rough sets and entropy measures," Journal of Biomedical Informatics, vol. 67, pp. 59-68, 2017.

[324] P. Maji and S. Paul, "Rough set based maximum relevancemaximum significance criterion and Gene selection from microarray data," International Journal of Approximate Reasoning, vol. 52, no. 3, pp. 408-426, 2011. 


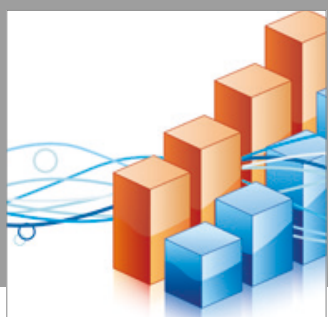

Advances in

Operations Research

vatersals

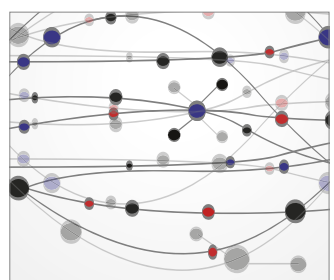

\section{The Scientific} World Journal
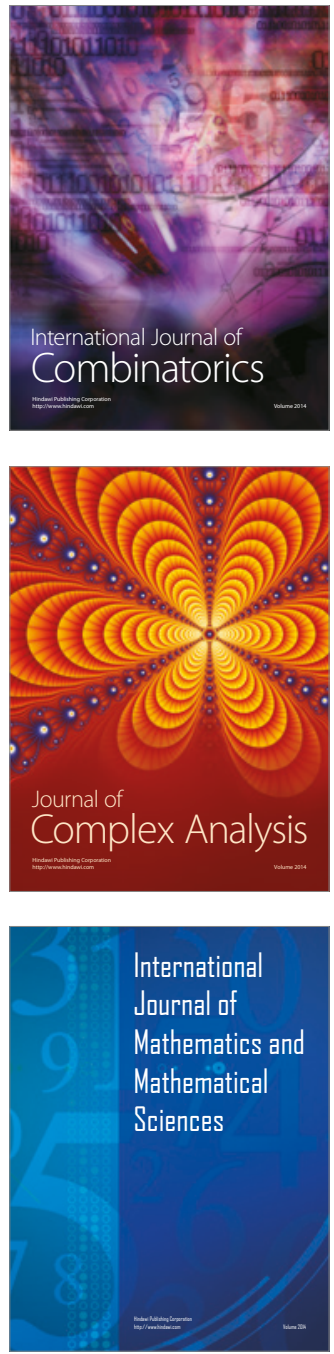
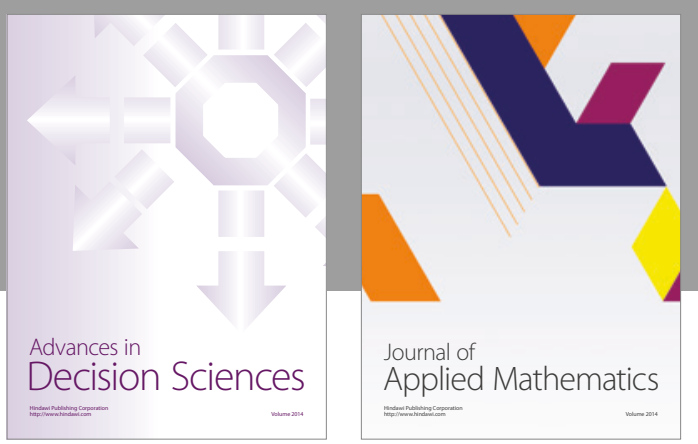

Algebra

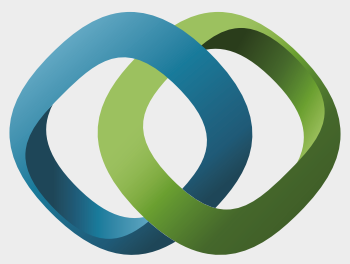

\section{Hindawi}

Submit your manuscripts at

https://www.hindawi.com
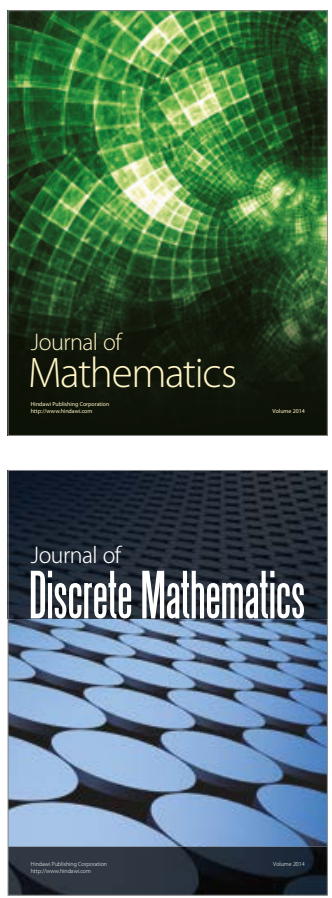

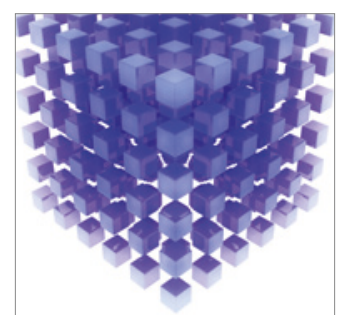

Mathematical Problems in Engineering
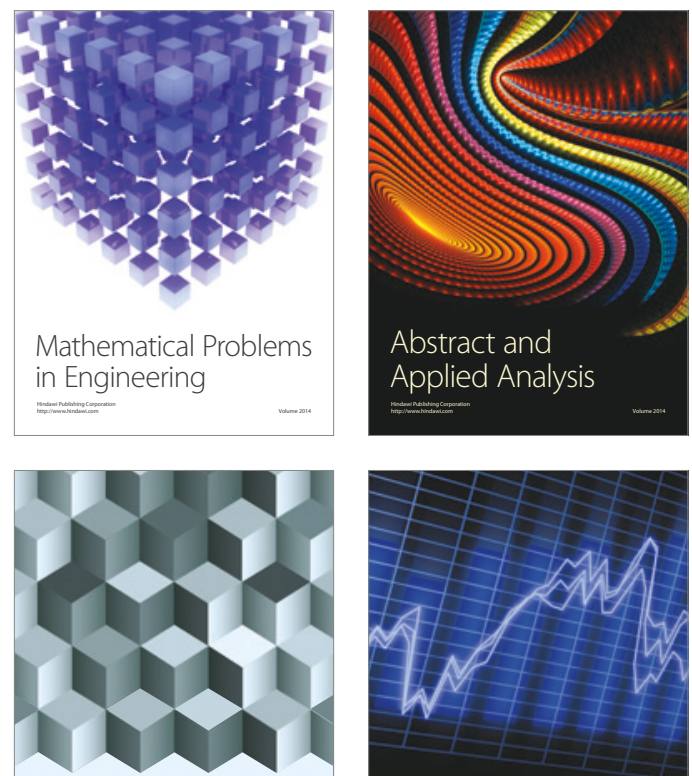

Journal of

Function Spaces

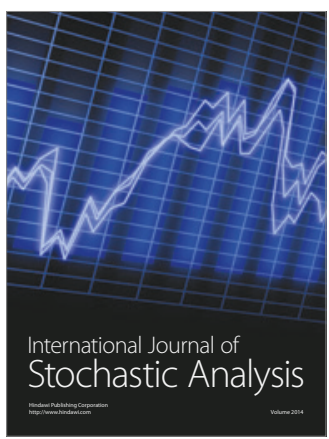

Probability and Statistics
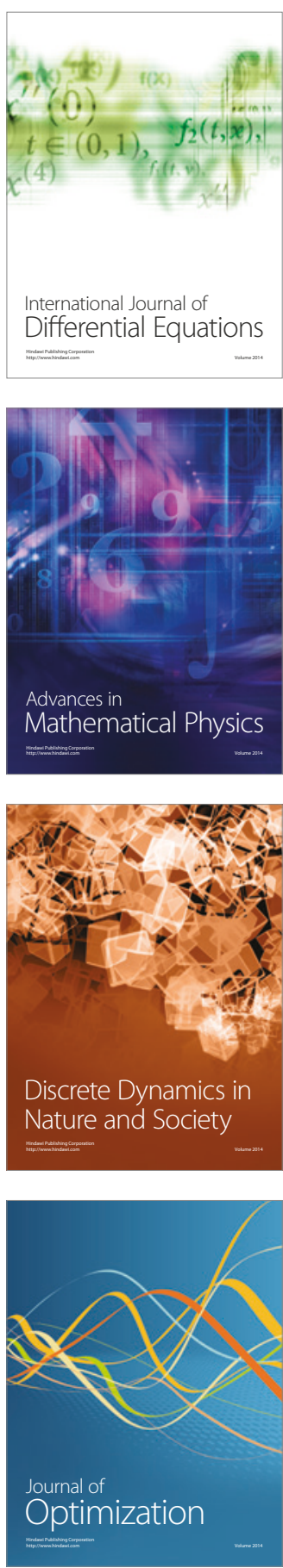